\title{
Host-directed therapy of tuberculosis based on interleukin-1 and type I interferon crosstalk
}

\author{
Katrin D. Mayer-Barber ${ }^{1}$, Bruno B. Andrade ${ }^{1}$, Sandra D. Oland ${ }^{1}$, Eduardo P. Amaral ${ }^{1,2}$, Daniel L. Barber ${ }^{3}$, Jacqueline Gonzales ${ }^{4}$, \\ Steven C. Derrick ${ }^{5}$, Ruiru Shi ${ }^{6}$, Nathella Pavan Kumar, ${ }^{7}$, , Wang Wei ${ }^{6}$, Xing Yuan ${ }^{6}$, Guolong Zhang ${ }^{9}$, Ying Cai ${ }^{4}$, Subash Babu ${ }^{7}, 10$, \\ Marta Catalfamo ${ }^{11}$, Andres M. Salazar ${ }^{12}$, Laura E. Via ${ }^{4}$, Clifton E. Barry III ${ }^{4} \&$ Alan Sher ${ }^{1}$
}

Tuberculosis remains second only to HIV/AIDS as the leading cause of mortality worldwide due to a single infectious agent ${ }^{1}$. Despite chemotherapy, the global tuberculosis epidemic has intensified because of HIV co-infection, the lack of an effective vaccine and the emergence of multi-drug-resistant bacteria ${ }^{2-5}$. Alternative host-directed strategies could be exploited to improve treatment efficacy and outcome, contain drug-resistant strains and reduce disease severity and mortality ${ }^{6}$. The innate inflammatory response elicited by Mycobacterium tuberculosis (Mtb) represents a logical host target ${ }^{7}$. Here we demonstrate that interleukin-1 (IL-1) confers host resistance through the induction of eicosanoids that limit excessive type I interferon (IFN) production and foster bacterial containment. We further show that, in infected mice and patients, reduced IL-1 responses and/or excessive type I IFN induction are linked to an eicosanoid imbalance associated with disease exacerbation. Host-directed immunotherapy with clinically approved drugs that augment prostaglandin E2 levels in these settings prevented acute mortality of Mtb-infected mice. Thus, IL-1 and type I IFNs represent two major counter-regulatory classes of inflammatory cytokines that control the outcome of Mtb infection and are functionally linked via eicosanoids. Our findings establish proof of concept for host-directed treatment strategies that manipulate the host eicosanoid network and represent feasible alternatives to conventional chemotherapy.

The mechanisms by which IL-1-driven inflammation promotes host resistance against $\mathrm{Mtb}$ in vivo have not been elucidated and offer novel targets for immunological intervention in tuberculosis (TB). Despite the high susceptibility of IL-1-deficient mice to Mtb infection, established mediators of host resistance, such as interferon (IFN) $-\gamma$, tumour necrosis factor (TNF)- $\alpha$, inducible nitric oxide synthase (iNOS) or IL12 p40 were not diminished in the absence of IL1R1 when assessed in vitro, in vivo at the single-cell level or in bronchoalveolar lavage fluid (BALF) (Extended Data Fig. 1a-c) $)^{8,9}$. Instead, we observed that increased bacterial loads in BALF were accompanied by an eicosanoid imbalance (Fig. 1a, b, Extended Data Fig. 1d). Eicosanoids are lipid mediators derived from arachidonic acid that include prostaglandins, resolvins, lipoxins and leukotrienes ${ }^{10}$. Cyclooxygenase-2 (COX-2, gene also known as Ptgs2) and 5-lipoxygenase (5-LO, gene also known as Alox5) or 12/ 15-lipoxygenase (12/15-LO) compete for arachidonic acid to generate COX-dependent prostaglandins or LO-dependent lipoxins and leukotrienes. Alox $5^{-1-}$ mice have been reported to be more resistant and prostaglandin E synthase (Ptges)-deficient mice more susceptible to Mtb infection $^{11-13}$. In the absence of IL-1 signalling, we found that prostaglandin E2 (PGE2) and prostaglandin F2 $\alpha$ (PGF2 $\alpha$ ) were significantly reduced in BALF of Mtb-infected $I l 1 r 1^{-/-}$mice, whereas the
5-LO products LXA4 and LTB4 were increased compared to wild-type animals (Fig. 1b, Extended Data Fig. 1d).

We then purified myeloid cells from infected wild-type lungs by fluorescence-activated cell sorting (FACS), and found that they produced large amounts of PGE2 ex vivo (Extended Data Fig. 1e). We next used mixed bone marrow chimaeras to analyse the requirement for IL1R1 on pulmonary CD68 ${ }^{\text {pos }}$ myeloid cells for COX-2 production in vivo. Il1 $\mathrm{r}^{-/-}$cells expressed significantly less COX-2 protein, indicating that IL-1 acts directly on myeloid cells for optimal COX-2 induction (Extended Data Fig. 1b, f) independently of pulmonary bacterial loads. In vitro, Mtb-infected Illr1 ${ }^{-1-}$ bone-marrow-derived macrophages (BMDM) failed to control bacterial growth compared to wild-type BMDM (Fig. 1c) and the increased colony forming units in $I l 1 r 1^{-1-}$ BMDM or bone-marrow dendritic cell cultures were associated with decreased PGE2 (Extended Data Fig. 2a, b). In addition, we observed a significant increase in both extracellular bacterial as well as infected macrophage numbers in Illa,Ill $b^{-1-}$ cultures (Extended Data Fig. 2c, d). Add-back of exogenous IL- $1 \alpha$, IL-1 $\beta$ or PGE2 reduced extracellular bacteria to wild-type levels (Fig. 1d, Extended Data Fig. 2e-g) and restored control of bacterial growth in IL-1-deficient BMDM (Fig. 1e and Extended Data Fig. 3a).

IL-1-dependent bacterial control was lost when COX-2 was inhibited with valdecoxib and restored upon PGE2 addition (Fig. 1e and Extended Data Fig. 3b). Of note, valdecoxib increased bacterial loads and inhibited PGE2 synthesis in infected wild-type BMDM as was observed in BMDM derived from $\mathrm{B} 6$ mice deficient in the enzymatic activity of COX-2 (Ptgs2(Y385F) mice) (Extended Data Fig. 3c). In vivo, absence of COX-2 enzymatic activity did not affect pulmonary and serum levels of PGE2 or lung bacterial loads in Ptgs2(Y385F) mice at 4 weeks after $\mathrm{Mtb}$ infection, consistent with a compensatory role for COX-1, which can generate PGE2 when COX-2 is absent ${ }^{14}$ (Fig. 1f, Extended Data Fig. 3d, e). Nonetheless, at later stages of Mtb infection, Ptgs2(Y385F) mice exhibited increased susceptibility compared to wild-type animals (Fig. 1g). We next examined whether IL-1 also drives PGE2 synthesis in infected primary human monocyte-derived macrophages (MDM). Blockade of IL1R1 signalling resulted in reduced PGE2 levels, whereas addition of exogenous IL- $1 \alpha$ or IL- $1 \beta$ further increased PGE2 synthesis during Mtb infection (Fig. 1h). Although additional PGE2-independent mechanisms of IL-1-mediated protection are likely to exist, our data demonstrate in vitro and in vivo that IL- $1 \alpha$ and IL- $1 \beta$, acting partially through COX-2, trigger PGE2 synthesis, which in turn regulates the containment and replication of bacilli during Mtb infection.

In many settings type I IFNs promote bacterial virulence and disease exacerbation ${ }^{15,16}$. Hypervirulence of certain Mtb strains correlates with

Immunobiology Section, Laboratory of Parasitic Diseases (LPD), National Institute of Allergy and Infectious Diseases (NIAID), National Institutes of Health (NIH), Bethesda, Maryland 20892, USA. ${ }^{2}$ Department of Immunology, Biomedical Sciences Institutes, University of Sao Paulo, 05508-900 Sao Paulo, Brazil. ${ }^{3} \mathrm{~T}$ Lymphocyte Biology Unit, LPD, NIAID, NIH, Bethesda, Maryland 20892, USA.

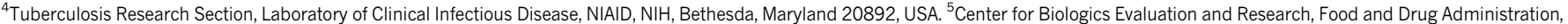

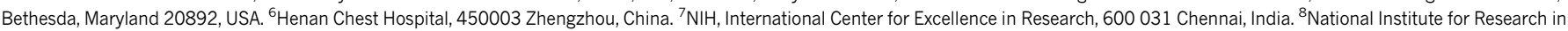

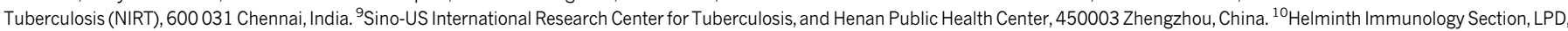

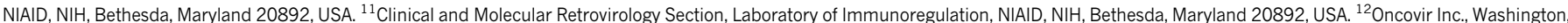
Washington DC 20008, USA 


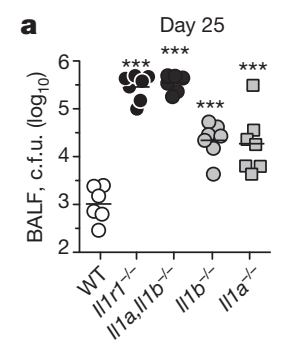

e
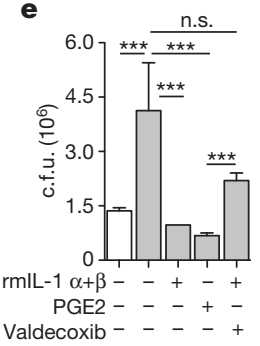
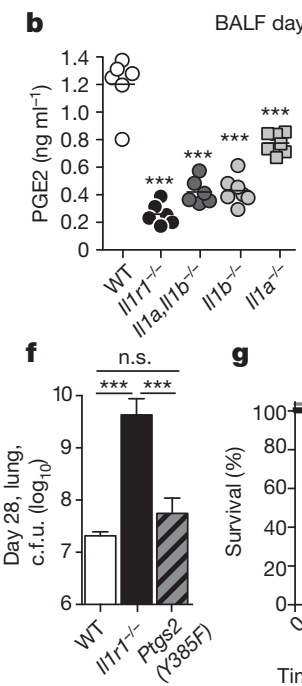

$\mathbf{g}$

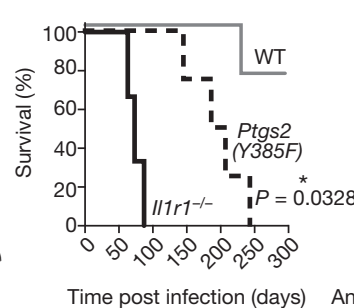

Time post infection (days)
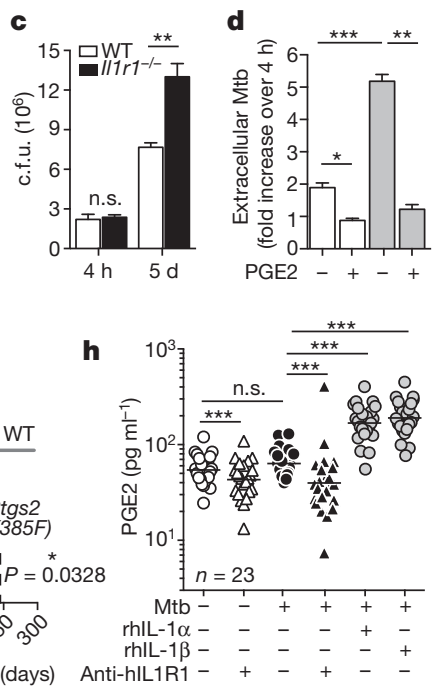

Figure $1 \mid$ IL-1 triggers PGE2 synthesis during Mtb infection. a, Bacterial loads (colony forming units, c.f.u.); b, PGE2 concentrations and PGE2/LXA ratio 25 days after aerosol exposure to Mtb in BALF of C57BL6 wild type (WT), Illr1 $1^{-/-}$, Ill $\alpha, I l 1 \beta^{-/-}, I l 1 \beta^{-1-}$ or Il1 $a^{-1-}$ mice $(n=4-8)$. c, c.f.u. in Mtbinfected (m.o.i. = 3) WT or Illr1 ${ }^{-\prime-}$ BMDM cultures. d, Mtb-RFP-infected (m.o.i. = 3) WT and Ill $\alpha, I l 1 \beta^{-1-}$ BMDMs cultured with or without PGE2. Extracellular bacteria were analysed by FACS (day 5). e, c.f.u. after in vitro infection (m.o.i. $=3$ ) of WT or Ill $\alpha, I l 1 \beta^{-1-}$ BMDM in the presence or absence of IL1, PGE2 or the COX-2 inhibitor valdecoxib. rmIL-1 $\alpha$, recombinant murine IL-1 $\alpha$. f, Lung c.f.u. of 4-week Mtb-infected WT, Illr1 $1^{-/-}$or Ptgs2(Y385F)

enhanced necrosis, type I IFN production and Mtb-infected Ifnar $1^{-/-}$ mice harbour fewer bacteria compared to wild-type animals ${ }^{8,17-19}$. Type I IFNs subvert anti-tuberculous host defences by inhibiting iNOS, mice $(n=5)$. a-f, Data are representative of two independent experiments. ${ }^{*} P \leq 0.05 ; * * P<0.005 ; * * * P<0.0005$; compared as indicated in figure by lines or to WT control groups. Error bars denote s.d. (MannWhitney test). g, Survival of WT, Illr1 $1^{-1-}$ or Ptgs2(Y385F) animals after aerosol challenge with 25-40 c.f.u. Data shown are from one experiment $(n=4$, Mantel-Cox test). h, PGE2 concentration in supernatants of MDM from 23 healthy donors, $24 \mathrm{~h}$ after Mtb infection in the presence or absence of rhIL- $1 \alpha$, rhIL- $1 \beta$ or anti-hIL1R1 neutralizing antibody. rhIL- $1 \alpha$, recombinant human IL- $1 \alpha$; anti-hIL1R1, anti-human IL-1R1. Differences were compared as indicated by lines (Wilcoxon matched pairs test).

IL-12p40, IL- $1 \alpha$ and IL-1 $\beta$ production, while inducing the immunosuppressive mediators IL-10 and IL1R antagonist (IL1Ra, gene also known as $(l 11 r n)^{8,19}$. Consistent with our previous findings that type I IFNs

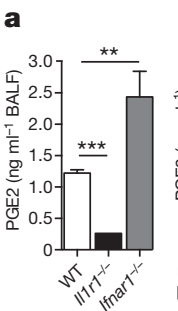

b
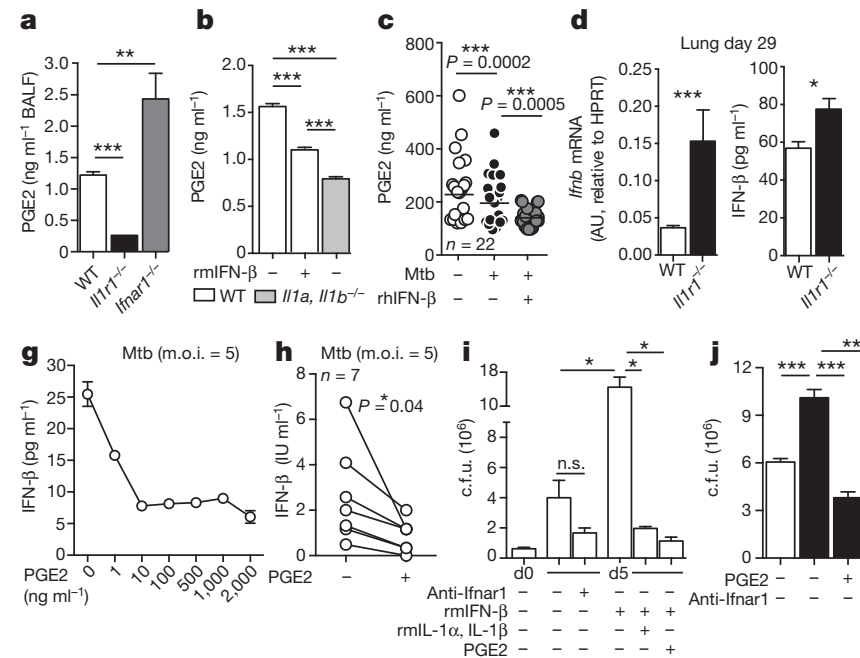

Figure $2 \mid$ IL-1 and PGE2 negatively regulate type I IFNs. a, b, PGE2 measured in BALF of indicated mouse strains 4 weeks post infection (p.i.) $(n=5)(\mathbf{a})$ and WT or Ill $\alpha, I l 1 \beta^{-l-}$ BMDM infected for $40 \mathrm{~h}(\mathrm{~m} . o . i .=5)$ or in the presence or absence of recombinant murine IFN- $\beta(\operatorname{rmIFN} \beta ; \mathbf{b}) . \mathbf{c}$, PGE2 in supernatants of MDM from 22 healthy donors, $24 \mathrm{~h}$ after Mtb infection $($ m.o.i. $=5)$ in the presence or absence of rhIFN- $\beta$ (Wilcoxon matched pairs test). d, Ifn $b$ mRNA expression and IFN- $\beta$ protein in lungs of Mtb-infected WT or Illr $1^{-/-}$mice at indicated time points. AU, arbitrary units $(n=9) . \mathbf{e}, \mathbf{f}, \mathrm{IFN}$ $\beta$ protein in supernatants of WT or Il1 $\alpha, I l 1 \beta^{-1-}$ BMDM infected for $40 \mathrm{~h}(\mathbf{e})$ or WT BMDM in the presence or absence of rmIL- $1 \alpha$ or IL- $1 \beta$ (f). $g$, IFN- $\beta$ protein concentration in supernatants WT BMDM $(40 \mathrm{~h}$ p.i. Mtb) treated with increasing concentrations of PGE2. h, IFN- $\beta$ protein in supernatants of

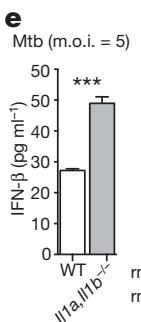

$\mathbf{f}$
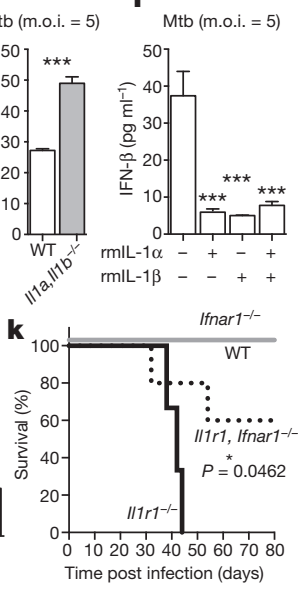

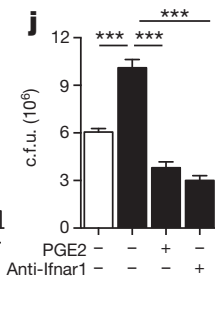

Time post infection (days)
MDM from 7 healthy donors, $24 \mathrm{~h}$ after Mtb infection with or without PGE2 (Wilcoxon matched pairs test). i, c.f.u. at indicated time points of Mtb-infected $($ m.o.i. $=5)$ WT BMDM cultures treated with anti-IFNAR1 monoclonal antibody, rmIL- $1 \alpha$ or Il- $1 \beta$, rmIFN- $\beta$ or PGE2. $\mathbf{j}$, c.f.u. after 5 days of Mtb infection (m.o.i. $=5$ ) of $I l 1 r 1^{-1-}$ BMDM treated with anti-mIFNAR1 $\mathrm{mAb}$ or PGE2. k, Survival of WT, Illr1 $1^{-1-}$, Ifnar $1^{-/-}$or Il1r1,Ifnar1 $1^{-1-}$ double-deficient animals after Mtb infection ( $n=5$, Mantel-Cox test). Data shown are representative of two $(\mathbf{a}, \mathbf{b}, \mathbf{d}-\mathbf{g}, \mathbf{i}, \mathbf{k})$ or three $(\mathbf{j})$ independent experiments. ${ }^{*} P \leq 0.05 ; * * P<0.005 ;{ }^{* * *} P<0.0005$; compared as indicated in figure by lines or to WT control groups. Error bars denote s.d. (Mann-Whitney test). 

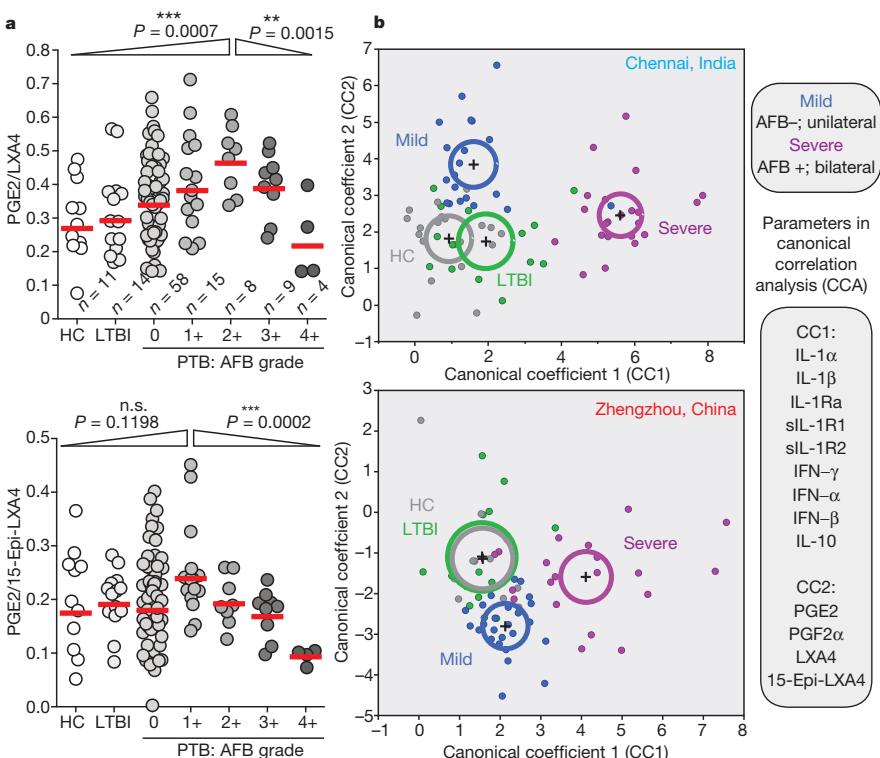

Figure $3 \mid$ IL-1, interferon and eicosanoid pathways are engaged in active TB disease. a, Mann-Kendall linear trend post hoc comparison of ratios of PGE2/LXA4 and PGE2/15-Epi-LXA4 according to AFB grade in the Chinese cohort. Each dot represents one individual with triangles depicting directionality of trend. b, PTB patients were stratified into mild (AFB-, unilateral lung lesions) and severe disease ( $\mathrm{AFB} \geq 1+$, and bilateral lung lesions) categories and canonical correlation analysis (CCA) was performed independently for Indian and Chinese cohorts. Indicated parameters were used to differentiate PTB from HC and LTBI groups as well as clinical severity within PTB group based on combined canonical coefficients. sIL-1R, soluble IL-1R.

antagonize the IL-1 pathway during Mtb infection ${ }^{8}$, absence of type I IFN signalling resulted in increased PGE2 and IL-1 $\beta$ and decreased IL1Ra in BALF (Fig. 2a and Extended Data Fig. 4a). Mtb-infected wildtype BMDM produced significantly less PGE2 when exogenous IFN- $\beta$ was present (Fig. 2b). Addition of IFN- $\beta$ to infected human MDM also significantly reduced PGE2 (Fig. 2c, Extended Data Fig. 4b), yet supplementation of IFN- $\beta$-treated cultures with IL-1 failed to restore PGE2 levels (Extended Data Fig. 4c). Together these observations suggested that, in addition to IL-1 itself ${ }^{8}$ and even in the presence of exogenous IL-1, type I IFNs potently counter-regulate the prostaglandin axis in Mtbinfected human and murine cells.

We next asked whether in the opposing direction IL-1 and PGE2 inhibit the type I IFN pathway, which could represent an important mechanism of IL-1/PGE2-dependent bacterial control. Indeed, in Mtbinfected $I l 1 r 1^{-/-}$mice pulmonary messenger RNA and protein levels of IFN- $\beta$ were significantly elevated compared to wild-type mice (Fig. $2 \mathrm{~d}$ ). Mtb-infected Illa,Il1 $b^{-l-}$ BMDM also expressed significantly elevated Ifna, Ifnb, IFN-inducible Il1rn (IL1Ra) and Il1r2 decoy receptor mRNA as well as IFN- $\beta$ protein (Extended Data Fig. $4 \mathrm{~d}$ and Fig. $2 \mathrm{e}$ ). Exogenous IL- $1 \alpha$ and IL- $1 \beta$ inhibited IFN- $\beta$ protein in wild-type macrophages and reduced mRNA expression in Illa,Ill $b^{-1-}$ BMDM (Fig. 2f, Extended Data Fig. 4e). Importantly, exogenous PGE2 also suppressed type I IFN production in Mtb-infected murine and human macrophages (Fig. 2g, h). Addition of either IL- 1 or PGE2 to IFN- $\beta$-treated BMDM cultures reversed IFN- $\beta$-dependent loss of bacterial control (Fig. $2 \mathrm{i}$ ). IL-1 failed to reverse PGE2 inhibition by type I IFNs, yet fully restored bacterial control, suggesting the existence of other IL-1-driven anti-mycobacterial mechanisms in addition to PGE2 regulation.

To investigate if the IL-1-type I IFN counter-regulatory pathway has an important function in IL-1-dependent bacterial control, we neutralized type I IFN in infected Illr1 ${ }^{-1-}$ BMDM. Blocking type I IFN signalling significantly reduced bacterial growth in $I l 1 r 1^{-1-}$ macrophages (Fig. 2j). Importantly, mice doubly deficient in both IL1R1 and IFNAR1
(Il1r1, Ifnar $1^{-/-}$) were significantly less susceptible to Mtb infection than Ill $r 1^{-1-}$ single-deficient animals (Fig. 2k). These findings reveal suppression of type I IFNs and their pro-bacterial activity as a major mechanism of IL-1- and PGE2-mediated host resistance against Mtb.

In previous studies both the development of active TB in latently infected individuals and the extent of radiographic disease among active TB patients were found to be closely associated with a type I IFN gene signature $\mathrm{e}^{20-22}$. Therefore we speculated that the IL-1-type I IFN balance might be particularly relevant in active TB patients with more severe disease presentations, than in latent or asymptomatic cases ${ }^{23}$. We retrospectively measured components of the IL-1, eicosanoid and IFN pathways in plasma of patients from Chennai, India, stratified into healthy controls (HC), latently infected individuals (LTBI) and pulmonary TB (PTB) cases based on their acid fast (AFB) sputum classification (Supplementary Tables $1,2,6)$. With increasing disease status, IL- $1 \alpha$ was reduced whereas PGE2, LXA4 and 15-Epi-LXA4 showed a significant trend to increase (Supplementary Table 6). We next measured the same parameters in a cohort from Zhengzhou, China, and found similar trends for IL-1 $\alpha$, PGE2 and 15-Epi-LXA4 (Supplementary Tables 3, 5, 7). The decreases in plasma IL-1 $\alpha$ observed here diverge from previous findings in BALF or alveolar macrophages of active TB patients, where IL$1 \beta$ was shown to be elevated ${ }^{24-27}$. This may reflect differences in systemic versus pulmonary cytokine levels or between the IL-1 species because we observed a similar dichotomy in mice infected with Mtb (Extended Data Fig. 5). The finding of increased PGE2 concentrations in PTB compared to HC and/or LTBI patients was unanticipated based on our murine data. Nevertheless, when we investigated the eicosanoid balance within the PTB group, as proposed above, we found a significant linear trend for PGE2/LXA4 or PGE2/15-Epi-LXA4 ratios to be decreased with higher sputum grades (Fig. 3a). We then further stratified the PTB group into patients with mild or severe disease (Fig. 3b, Supplementary Table 5). Importantly, in both study sites the IL-1, eicosanoid and IFN pathways faithfully distinguished PTB patients based on disease severity after canonical correlation analysis (CCA) ${ }^{28}$ (Fig. 3b, Supplementary Table 8). Thus, clinical data from two independent study cohorts support the hypothesis that TB severity is associated with changes in IL-1, type I IFNs and eicosanoids thus supporting a link between these pathways in active tuberculosis.

On the basis of these observations we proposed that a host-directed therapy (HDT) targeting eicosanoids would be most effective in active disease associated with necrotic lung pathology where high type I IFN conditions dominate. Indeed, when Illa, Ill $b^{-}{ }_{-}^{-}$animals were administered PGE2 together with zileuton, a 5-LO inhibitor (Extended Data Fig. 6a), pulmonary bacterial loads and pathology were reduced and survival enhanced (Fig. 4a, Extended Data Fig. 6b-e). Of note, when the same treatment was given to wild-type animals for up to 6 months bacterial control was not enhanced nor were negative side effects observed (Fig. 4a, Extended Data Fig. 6b, e and data not shown). Importantly, PGE2 and zileuton treatment had no effect on survival of susceptible Ifng $^{-/-}$or Tnfa $a^{-1-}$ animals (Extended Data Fig. 6f). Thus, the observed treatment is specific for IL-1-mediated host resistance, rather than owing to indirect effects on pathways generally shared by susceptible mice. We next administered either PGE2 or zileuton alone and found that add-back of PGE2 extended survival of $I l 1 r 1^{-1-}$ animals, whereas administration of zileuton failed to do so (Extended Data Fig. 6g). Consistent with the latter finding, infected Il1r1,Alox $5^{-1-}$ mice died with similar kinetics as $I l 1 r 1^{-1-}$ animals (Extended Data Fig. 6g). These data indicate that the susceptibility of IL-1-deficient mice is linked to decreased PGE2, rather than to an increase in 5-LO products, and that yet unidentified functions of IL-1 contribute to host resistance against $\mathrm{Mtb}$, because rescue of IL-1-deficient animals, either by PGE2 addback or type I IFN neutralization, was incomplete.

We then tested the eicosanoid-based HDT in a model using IL-1replete animals where induction of high type I IFN levels by intranasal administration of polyinosinic-polycytidylic acid stabilized with polyL-lysine (pICLC) causes uncontrolled disease leading to mortality and 
a

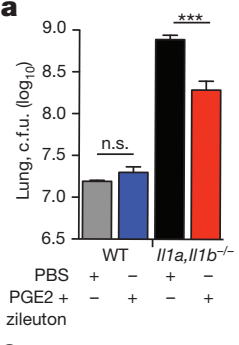

c
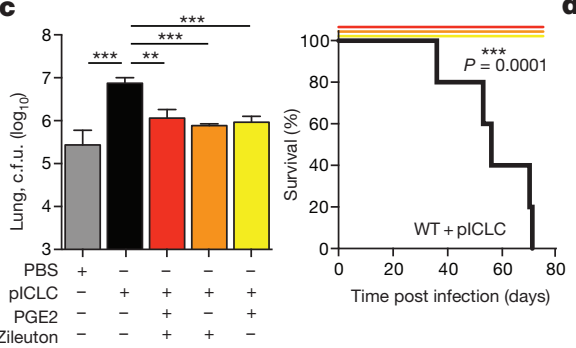

Figure 4 HDT targeting eicosanoids confers protection in highly susceptible mice associated with high type I IFN responses. a, Lung c.f.u. (day 21 p.i.) (left, $n=10$, error bars denote s.d., Mann-Whitney test) and survival (right, $n=13$, Mantel-Cox test between treated and untreated $I l 1 \alpha, I l 1 \beta^{-1-}$ mice) after aerosol exposure to Mtb of WT or Ill $\alpha, I l 1 \beta^{-1-}$ treated with or without intranasal (i.n.) PGE2 (twice a week) and zileuton (in drinking water) starting day 1 p.i. Data shown are combined from two independent experiments. b-e, Mice were treated i.n. with pICLC or without (PBS) twice a week, starting day 1 after aerosol challenge with Mtb. b. Weight loss (left, travelling error bars indicate s.d., $n=7$ ) and survival (right) during Mtb infection of WT (pICLC or PBS i.n.) mice treated with or without i.n. PGE2 and zileuton. c, Lung c.f.u. 4 weeks p.i. of WT mice treated as indicated (left, $n=5$, error bars denote s.d. (Mann-Whitney test). Survival (right) of
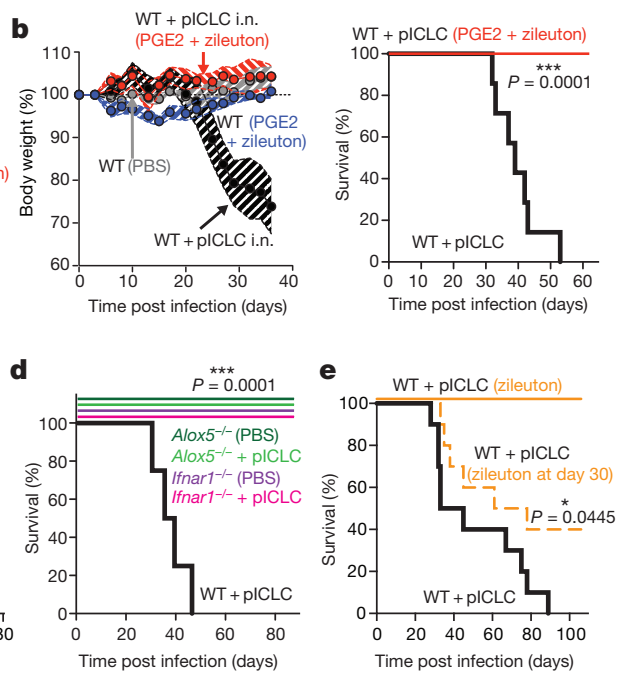

pICLC-treated WT mice (black) compared to those who also received PGE2 (yellow), zileuton (orange) or both (red). Differences were compared to pICLC group (Mantel-Cox test). Data are representative of three independent experiments $(n=5)$. d, Survival of pICLC-treated WT mice (black), Ifnar $1^{-/-}$ animals (purple, without pICLC in pink) or Alox $5^{-/-}$mice (dark green, without pICLC in light green). Statistical significance was compared to pICLC group. Data are representative of two independent experiments $(n=4-6)$. e, Survival of pICLC-administered WT mice treated with zileuton in drinking water starting day 1 (solid orange line) compared to $\mathrm{d} 30$ (dotted orange line). Data shown are combined from two independent experiments $(n=10)$. Statistical significance was compared to pICLC (black) group with Mantel-Cox test. ${ }^{*} P \leq 0.05$; ${ }^{*} P<0.005$; ${ }^{* * *} P<0.0005$.

mortality, it is possible that the HDT protects via PGE2-mediated bacterial control and/or inhibition of type I IFNs rather than suppression of 5 - $\mathrm{LO}$ products.

Finally, we tested the therapeutic efficacy of 5-LO blockade by initiating Zileuton treatment at 30 days post-infection, when PICLC-treated animals already display $10-20 \%$ weight loss (Fig. 4 e and data not shown). This resulted in enhanced survival, with $40 \%$ of the animals remaining alive at the end of the study. Thus, manipulation of the eicosanoid balance towards PGE2 can both prevent and therapeutically ameliorate disease exacerbation associated with type I IFN expression (Extended Data Fig. 10). Although eicosanoids have been suggested previously as targets for therapeutic intervention in tuberculosis, the data presented here provide in vivo proof of concept for this approach in a mammalian model of pulmonary Mtb infection.

\section{METHODS SUMMARY}

Eight-to-twelve-week-old C57BL/6 or gene-deficient animals were aerosol infected with Mtb strain H37Rv (100-150 colony forming units per mouse unless stated otherwise). In vitro infections of MDM or BMDM were performed at indicated multiplicity of infection (m.o.i.). Eicosanoids and cytokines were measured between 24 and $48 \mathrm{~h}$ after in vitro, in vivo at indicated time points or in plasma of TB patients using commercial enzyme immunoassay (EIA) or enzyme-linked immunosorbent assay (ELISA) kits. For in vitro colony forming units determination, supernatants were collected day 5 post infection and cells lysed in distilled $\mathrm{H}_{2} \mathrm{O}$ for $5-10 \mathrm{~min}$. Supernatants and cell lysate were then combined to evaluate total mycobacterial growth. Extracellular colony forming units were determined by microscopy or flow cytometry as described in Methods. Antibodies, PCR primers and other pharmacological reagents as well as staining, RT-PCR and cell differentiation protocols are listed in Methods. C57BL/6 mice were administered pICLC intranasally twice a week ( $6 \mu \mathrm{g}$ in $30 \mu \mathrm{l})$. For HDT mice were given PGE2 intranasally twice a week ( $2 \mu \mathrm{g}$ in $30 \mu \mathrm{l})$ or zileuton in drinking water ad libitum $\left(6 \mathrm{mg} \mathrm{ml}^{-1}\right)$ or both starting 1 day after infection, unless stated otherwise. Patient cohort descriptions and immune mediator measurements are provided in Supplementary Information and Methods. 
Online Content Methods, along with any additional Extended Data display items and Source Data, are available in the online version of the paper; references unique to these sections appear only in the online paper.

\section{Received 28 September 2013; accepted 16 May 2014.}

Published online 25 June 2014.

1. WHO. Tuberculosis Fact sheet $\mathrm{N}{ }^{\circ} 104$. http://www.who.int/mediacentre/ factsheets/fs104/en/index.html (2012).

2. Russell, D. G., Barry, C. E. III \& Flynn, J. L. Tuberculosis: what we don't know can, and does, hurt us. Science 328, 852-856 (2010)

3. Bishai, W., Sullivan, Z., Bloom, B. R. \& Andersen, P. Bettering BCG: a tough task for a TB vaccine? Nature Med. 19, 410-411 (2013).

4. Lawn, S. D. \& Zumla, A. I. Tuberculosis Lancet 378, 57-72 (2011).

5. Gandhi, N. R. etal. Multidrug-resistant and extensively drug-resistant tuberculosis: a threat to global control of tuberculosis. Lancet 375, 1830-1843 (2010).

6. Nathan, C. Fresh approaches to anti-infective therapies. Sci. Transl. Med. 4, $140 \mathrm{sr} 142(2012)$

7. Kaufmann, S. H. \& Dorhoi, A. Inflammation in tuberculosis: interactions, imbalances and interventions. Curr. Opin. Immunol. 25, 441-449 (2013).

8. Mayer-Barber, K. D. et al. Innate and adaptive interferons suppress IL- $1 \alpha$ and IL-1 $\beta$ production by distinct pulmonary myeloid subsets during Mycobacterium tuberculosis infection. Immunity 35, 1023-1034 (2011).

9. Mayer-Barber, K. D. et al. Caspase-1 independent IL-1 $\beta$ production is critical for host resistance to Mycobacterium tuberculosis and does not require TLR signaling in vivo. J. Immunol. 184, 3326-3330 (2010).

10. Serhan, C. N., Chiang, N. \& Van Dyke, T. E. Resolving inflammation: dual antiinflammatory and pro-resolution lipid mediators. Nature Rev. Immunol. 8, 349-361 (2008).

11. Bafica, A. et al. Host control of Mycobacterium tuberculosis is regulated by 5-lipoxygenase-dependent lipoxin production. J. Clin. Invest. 115, 1601-1606 (2005).

12. Chen, M. et al. Lipid mediators in innate immunity against tuberculosis: opposing roles of PGE2 and LXA4 in the induction of macrophage death. J. Exp. Med. 205, 2791-2801 (2008)

13. Divangahi, M. et al. Mycobacterium tuberculosis evades macrophage defenses by inhibiting plasma membrane repair. Nature Immunol. 10, 899-906 (2009).

14. Kirtikara, K. et al. Compensatory prostaglandin E2 biosynthesis in cyclooxygenase 1 or 2 null cells. J. Exp. Med. 187, 517-523 (1998).

15. Antonelli, L. R. et al. Intranasal Poly-IC treatment exacerbates tuberculosis in mice through the pulmonary recruitment of a pathogen-permissive monocyte/ macrophage population. J. Clin. Invest. 120, 1674-1682 (2010).

16. Rayamajhi, M., Humann, J., Kearney, S., Hill, K. K. \& Lenz, L. L. Antagonistic crosstalk between type I and II interferons and increased host susceptibility to bacterial infections. Virulence 1, 418-422 (2010).

17. Manca, C. et al. Virulence of a Mycobacterium tuberculosis clinical isolate in mice is determined by failure to induce Th1 type immunity and is associated with induction of IFN- $\alpha / \beta$. Proc. Natl Acad. Sci. USA 98, 5752-5757 (2001)

18. Stanley, S. A., Johndrow, J. E., Manzanillo, P. \& Cox, J. S. The type I IFN response to infection with Mycobacterium tuberculosis requires ESX-1-mediated secretion and contributes to pathogenesis. J. Immunol. 178, 3143-3152 (2007)

19. O'Garra, A. et al. The immune response in tuberculosis. Annu. Rev. Immunol. 31, 475-527 (2013).

20. Berry, M. P. et al. An interferon-inducible neutrophil-driven blood transcriptional signature in human tuberculosis. Nature 466, 973-977 (2010).

21. Maertzdorf, J. et al. Human gene expression profiles of susceptibility and resistance in tuberculosis. Genes Immun. 12, 15-22 (2011).
22. Ottenhoff, T. H etal. Genome-wide expression profiling identifies type 1 interferon response pathways in active tuberculosis. PLOS ONE 7, e45839 (2012).

23. Barry, C. E. III et al. The spectrum of latent tuberculosis: rethinking the biology and intervention strategies. Nature Rev. Microbiol. 7, 845-855 (2009)

24. Law, K. et al. Increased release of interleukin-1 beta, interleukin-6, and tumor necrosis factor-alpha by bronchoalveolar cells lavaged from involved sites in pulmonary tuberculosis. Am. J. Respir. Crit. Care Med. 153, 799-804 (1996).

25. Tsao, T. C. et al. Increased TNF-alpha, IL-1 beta and IL- 6 levels in the bronchoalveolar lavage fluid with the upregulation of their mRNA in macrophages lavaged from patients with active pulmonary tuberculosis. Tuber. Lung. Dis. 79, 279-285 (1999)

26. Kuo, H. P. etal. Nitric oxide modulates interleukin-1 beta and tumor necrosis factoralpha synthesis by alveolar macrophages in pulmonary tuberculosis. Am. J. Respir. Crit. Care Med. 161, 192-199 (2000).

27. Wang, C. H. \& Kuo, H. P. Nitric oxide modulates interleukin-1beta and tumour necrosis factor-alpha synthesis, and disease regression by alveolar macrophages in pulmonary tuberculosis. Respirology 6, 79-84 (2001).

28. Rousu, J., Agranoff, D. D., Sodeinde, O., Shawe-Taylor, J. \& Fernandez-Reyes, D. Biomarker discovery by sparse canonical correlation analysis of complex clinical phenotypes of tuberculosis and malaria. PLOS Comput. Biol. 9, e1003018 (2013)

29. Tobin, D. M. et al. The Ita $4 \mathrm{~h}$ locus modulates susceptibility to mycobacterial infection in zebrafish and humans. Cell 140, 717-730 (2010).

30. Tobin, D. M. et al. Host genotype-specific therapies can optimize the inflammatory response to mycobacterial infections. Cell 148, 434-446 (2012).

Supplementary Information is available in the online version of the paper.

Acknowledgements This work was supported by the NIAID Intramural Research program and a Concept Acceleration Program-Award (K.D.M.-B., B.B.A. and A.S.) from DMID, NIAID. We are grateful to K. Elkins, S. Morris, M. Belcher as well as the NIAID ABSL3 support staff for facilitating our animal studies. We thank R. Chen, L. Goldfeder and Q. Gao for sharing their clinical trial expertise and research facilities, respectively. We also thank K. Kauffman, R. Thompson, S. Hieny, P. Dayal, D. Surman, L. Meng, Z. Li, L. Lifa, Q. Shen and Z. Huang for technical assistance, H. Boshoff for help with direct anti-mycobacterial activity assays and M. S. Jawahar, V.V. Banurekha and R. Sridhar for recruitment and clinical evaluation of patients in Chennai, India. We are grateful to F. Andrade Neto, H. Remold, K. Arora, J. Aliberti, M. Moayeri, P. Murphy, A. O'Garra, R. Germain and C. Serhan for discussion or critical reading of the manuscript. Finally, we thank the patients, volunteer participants, and clinical staff of the Tuberculosis department of Henan Chest Hospital in Zhengzhou, China and the Department of Clinical Research (NIRT) and Department of Thoracic Medicine (Government Stanley Medical Hospital) in Chennai, India for their participation in our clinical studies.

Author Contributions K.D.M.-B. conceived the study, designed and performed experiments, analysed data and wrote the paper; B.B.A. performed experiments, analysed data and prepared the Indian cohort description; E.P.A. and D.L.B. performed experiments; S.D.O., J.G., S.C.D., N.P.K., Y.C., L.E.V., provided technical or analytical assistance; S.B. recruited, sampled and collected data about patients and provided access to samples from Indian cohort, M.C. provided healthy donor material, A.M.S provided Hiltonol (pICLC); R.S., W.W., X.Y., G.Z., L.E.V. and C.E.B. conducted the Natural History Study in Zhengzhou, provided access to Chinese patient samples and the preparation of the Chinese cohort description, A.S. provided conceptual advice and wrote the paper and all authors approved the final manuscript.

Author Information Reprints and permissions information is available at www.nature.com/reprints. The authors declare no competing financial interests. Readers are welcome to comment on the online version of the paper. Correspondence and requests for materials should be addressed to K.D.M.-B. (mayerk@niaid.nih.gov) 


\section{METHODS}

Mice and Mtb infections. C57BL/6 mice were purchased from Taconic Farms (Hudson, NY). Illa ${ }^{-1-}, I l 1 b^{-1-}, I l 1 a, I l 1 b^{-1-}$ mice were originally derived by Y. Iwakura (Tokyo University). Illr1 ${ }^{-1-}, \operatorname{Ptgs} 2(\mathrm{Y} 385 \mathrm{~F}), \operatorname{Tnfa}^{-1-}$, Alox $^{-1-}$, Alox12/ $15^{-/-}, L t b 4 r 1^{-/-}$and $\mathrm{C} 3 \mathrm{HeB} / \mathrm{FeJ}$ mice were purchased from Jackson Laboratories (Bar Harbour, ME) and Illr1 $1^{-1-}$ mice were backcrossed to $\mathrm{B} 6$ for at least 10 generations. B6.SJL (CD45.1/1), B6.SJL (CD45.1/2), Il10 ${ }^{-1-}$, Ifng ${ }^{-/-}$, Ifnar1 ${ }^{-/-}$mice were obtained through a supply contract between the National Institute of Allergy and Infectious Diseases (NIAID) and Taconic Farms. Il1r1,Ifnar1 ${ }^{-1-}$ and Illr1, Alo $\times 5^{-1-}$ mice were generated by inter-breeding of Ill $11^{-1-}$ with Ifnar $1^{-1-}$ or Alo $\times 5^{-1-}$ mice. All animals were maintained in an AALAC-accredited BSL2 or BSL3 facilities at the NIH or FDA and experiments performed in compliance with an animal study proposal approved by the NIAID or FDA Animal Care and Use Committee. 8-12 weeks old male and female mice were used. Aerosol infection with the H37Rv strain of Mtb (100-150 c.f.u. per mouse unless stated otherwise) and determination of lung bacterial growth were performed as previously described ${ }^{9}$. Macrophage/dendritic cell differentiation and in vitro cultures. Murine bonemarrow cells were cultured for 7 days in either 15\% GM-CSF media to generate BMDC or 30\% L929 supernatant media to differentiate BMDM and then exposed to $\mathrm{H} 37 \mathrm{Rv}$ at an indicated multiplicity of infection (m.o.i.) for $24-48 \mathrm{~h}$ and supernatants harvested. In some experiments PGE2 $\left(2 \mu \mathrm{g} \mathrm{ml}^{-1}\right.$, Sigma), zileuton $(20 \mu \mathrm{M}$, Tocris), valdecoxib $\left(0.5 \mu \mathrm{M}\right.$, Tocris) or recombinant murine IFN $\beta\left(20 \mathrm{ng} \mathrm{ml}^{-1}\right)$, IL- $1 \alpha$ or IL-1 $\beta\left(10 \mathrm{ng} \mathrm{ml}^{-1}\right)$ or both together $\left(5 \mathrm{ng} \mathrm{ml}^{-1}+5 \mathrm{ng} \mathrm{ml}^{-1}, \mathrm{R} \& D\right.$ Systems, Minneapolis, MN) were added to the cultures.

Human elutriated monocytes were obtained from peripheral blood of healthy cytomegalovirus-negative donors. Monocyte derived macrophages (MDM) were generated by culturing monocytes with media containing M-CSF $\left(60 \mathrm{ng} \mathrm{ml}^{-1}\right)$ for 7 days. Fresh media with indicated growth factors was added every $48 \mathrm{~h}$. Cells were exposed to H37Rv (m.o.i. = 5) in the presence or absence of PGE2 $\left(2 \mu \mathrm{g} \mathrm{ml}^{-1}\right.$, Sigma), recombinant human IFN- $\beta\left(10 \mathrm{ng} \mathrm{ml}^{-1}\right), \mathrm{IL}-1 \alpha\left(10 \mathrm{ng} \mathrm{ml}^{-1}\right), \mathrm{IL}-1 \beta\left(10 \mathrm{ng} \mathrm{ml}^{-1}\right)$ or neutralizing anti-hIL1-R1 antibody $\left(20 \mu \mathrm{g} \mathrm{ml}^{-1}, \mathrm{R} \& \mathrm{D}\right.$ Systems, Minneapolis, MN) for $24 \mathrm{~h}$. All recombinant human cytokines were from Peprotech (Rocky Hill, NJ). Cytokine and eicosanoid measurements in culture supernatants and biological fluids. Murine and human cytokine and lipid mediator concentrations in culture supernatants were quantified using commercial ELISA kits (R\&D Systems) and enzyme immunoassay (EIA) kits (Cayman Chemical, Ann Harbour, MI and Oxford Biomedical Research, Oxford, MI). Murine bronchoalveolar lavage (BAL) fluid and cell free lung homogenates were obtained and cytokines and nitric oxide measured as described previously ${ }^{9}$. Eicosanoids in murine serum, BAL and lung were measured with EIA kits (Cayman Chemical, Ann Harbour, MI and Oxford Biomedical Research, Oxford, MI).

For clinical studies in India, plasma levels of human IL- $1 \alpha$, IL-1 $\beta$, IL-10, IL1Ra, IL1RI, IL1RII, IFN- $\gamma$, IFN- $\alpha$, IFN- $\beta$ and TNF- $\alpha$ were measured using commercial ELISA kits (R\&D Systems, Minneapolis, MN). Levels of PGE2, PGF2 $\alpha$ (Cayman Chemical, Ann Harbour, MI), LXA4 and 15-EPI-LXA4 (Oxford Biomedical Research, Oxford, MI), were assessed using EIA kits following the manufacturers' instructions. For clinical studies in China, plasma levels of IL-1 $\alpha$, IL-1 $\beta$, IL-10, IL1Ra, IL1RI, IL1RII, IFN- $\gamma$, IFN- $\alpha$, IFN- $\beta$ and TNF- $\alpha$ were measured using commercial ELISA kits (R\&D Systems, Minneapolis, MN) and Flowcytomix Multiplex Arrays (eBioscience, San Diego, CA) according to the manufacturer's instructions. Levels of PGE2, PGF2 $\alpha$, LXA4 and 15-EPI-LXA4 were assessed using EIA kits according to the manufacturer's directions (Oxford Biomedical Research, Oxford, MI). Flow cytometry. Abs against mouse surface antigens and cytokines were purchased from Ebioscience, Biolegend (San Diego, CA), AbD Serotec (Raleigh, NC) and BD Pharmingen (San Diego, CA) and used in 12-colour flow cytometry either biotinylated or directly conjugated. The Abs used were directed against Ly6C (clone AL21), Ly6G (1A8), CD11c (HL3 and N418), CD68 (FA-11), CD45.1 (A20), CD45.2 (104), TCRb (H57-597), NK1.1 (PK136), IL-1 $\alpha$ PE (ALF-161), IL-1 $\beta$ APC (pro-IL$1 \beta$ monoclonal NJTEN3), iNOS (polyclonal rabbit unconjugated), IL-12/23 p40 (C15.6), TNF- $\alpha$ (MP6-XT22), CD11b (M1/70), activated caspase-3 (BD Pharmingen), COX-2 (polyclonal rabbit unconjugated, Cell Signaling). Biotinylated and unconjugated polyclonal rabbit antibodies were detected with streptavidin-conjugated Qdot 605, or goat anti-rabbit secondary-AF647, respectively from Molecular ProbesInvitrogen (Carlsbad, Ca). Ultraviolet fixable live/dead cell stain was purchased from Molecular Probes-Invitrogen and used according to the manufacturer's protocol. All samples were acquired on a LSRII flow cytometer (BD Biosciences, San Jose, CA) and analysed using FlowJo software (Three Star, Ashland, OR).

Isolation and re-stimulation of myeloid cells from lung tissue. Perfused lungs from infected mice were cut into $1-2 \mathrm{~mm}$ pieces and subsequently digested with liberase $\mathrm{Cl}\left(0.4 \mathrm{mg} \mathrm{ml}^{-1}\right.$ in PBS; Roche-Diagnostics, Indianapolis, IN). The reaction was stopped after 30-45 min. with an equal volume of fetal calf serum. Digested lung was fully dispersed by passage through a $100-\mu \mathrm{m}$ pore-size cell strainer and an aliquot removed for bacterial load measurements. Isolated cells were then washed, counted and subsequently surface stained for FACS sorting in a BSL3 containment facility under sterile conditions. In some experiments FACS-sorted cells or wholelung single-cell suspensions from infected mice were stimulated overnight in the presence or absence of live $\mathrm{Mtb} \mathrm{H} 37 \mathrm{Rv}$ (m.o.i. $=1$ ) at $10^{6}$ cells per well in 96-well plates. Supernatants were then examined for lipid mediators or cytokines. In other experiments, infected lung cells were re-suspended in media containing monensin $\left(0.1 \%\right.$, Ebioscience) in the presence or absence of $100 \mu \mathrm{g} \mathrm{ml}^{-1}$ irradiated Mtb H37Rv at $10^{6}$ cells per well in 96 -well plates and incubated at $37^{\circ} \mathrm{C}, 5 \% \mathrm{CO}_{2} .5 \mathrm{~h}$ later cells were surface-stained, fixed/permeabilized and intracellular staining (ICS) performed. Preparation of mixed bone-marrow chimaeric mice. B6.SJL (CD45.1/1) mice were lethally irradiated (950 rad) and reconstituted with a total of $10^{7}$ donor bonemarrow cells from C57BL/6 CD45.1/2 wild-type (WT) mice mixed at equal parts with bone-marrow cells from mice deficient (KO) in CD45.2/2 $\mathrm{Illr} \mathrm{rl}^{-1-}$. Mice were allowed to reconstitute for 8-10 weeks before aerosol infection with H37Rv.

In vitro c.f.u. assay. $B M D M$ were infected with virulent $H 37 \mathrm{Rv}$ at indicated m.o.i. At indicated time points, supernatants were collected and cells lysed in distilled $\mathrm{H}_{2} 0$ for 5-10 min. Supernatants and cell lysate were combined to evaluate total mycobacterial growth by plating on Middlebrook 7H10 agar plates and incubating at $37^{\circ} \mathrm{C}$ for 21 days, after which c.f.u. were enumerated.

In vitro extracellular c.f.u. assay. $B M D M$ were infected with virulent $\mathrm{H} 37 \mathrm{Rv}$ at m.o.i. $=3$. At indicated time points, supernatants were collected and cells trypsinized for 5-10 min. Supernatants and cell lysates were combined and acid-fast-stained cytospin preparations were used to visualize and count intracellular AFB by light microscopy. A minimum of 400-500 macrophages, number of bacteria per macrophage and percentage of infected macrophages as well as cell-free bacteria, were counted by microscopy. Alternatively, BMDM were infected with virulent Mtb $\mathrm{H} 37 \mathrm{Rv}$ expressing the red fluorescent protein (H37Rv-RFP) (gift from J. Ernst) at m.o.i. $=3$. At indicated time points, supernatants were collected and cells trypsinized for 5-10 min. Supernatants and cell lysates were combined to evaluated extracellular and intracellular mycobacterial growth by acquiring the samples on an LSRII inside a BSL3 containment laboratory.

RT-PCR. Total RNA was isolated from cell suspension using the RNeasy mini kit (Qiagen, Valencia, CA). Briefly, cells were lysed in $400 \mu$ l of RLT buffer containing 2-mercaptoethanol. Lysates were then passed through QIA Shredder columns and RNA isolated on Qiagen mini columns (Qiagen), according to the manufacturer's protocol. Total RNA from lung tissue was isolated by homogenizing the lung tissue in TRIZOL (Life Technologies) and then using the Qiagen RNAeasy mini kit. Individual RNA samples (100 ng-1 $\mu$ g each) were reverse-transcribed using Superscript II (Invitrogen Life Technologies) and a mixture of oligo(dT) and random primers. Real-time PCR was performed on an ABI Prism 7900 sequence detection system (Applied Biosystems). Relative quantities of mRNA for several genes were determined using SYBR Green PCR Master Mix (Applied Biosystems) and by the comparative threshold cycle method, as described by Applied Biosystems for the ABI Prism 7700/7900 sequence detection systems. In this method, mRNA levels for each sample were normalized to hypoxanthine-guanine phosphoribosyl transferase (HPRT) mRNA levels and then expressed as a relative increase or decrease in arbitrary units (AU). Primers were designed using Roche Universal Probe Library (Roche). The sequences of the specific primers are as follows: Hprt: forward, cctcc tcagaccgcttttt; reverse, aacctggttcatcatcgctaa; Il1 r2: forward, tctggtacctacatttgcacattc; reverse, aaagaccttgagttccacagaca; Ill $r n$ : forward, tgtgccaagtctggagatga; reverse, tt ctttgttcttgctcagatcagt; Ifna: forward, aagcctgtgtgatgcagga; reverse, caggggctgtgtttc ttctc; Ifnb: forward, ggaaagattgacgtgggaga; reverse, cctttgcaccctccagtaat.

Histopathology. Lungs were fixed by inflating the tissues with $4 \%$ formaldehyde, sectioned and stained with haematoxylin and eosin or by the Ziehl-Neelsen method to visualize acid-fast mycobacteria. The extent of the pulmonary inflammatory response and was determined under light microscopy at magnifications of $\times 20$ and $\times 100$, respectively. The extent of necrosis in lungs was assessed microscopically using a scale of $1-5$, with 5 representing the most severe necrotic response. All histological analyses were performed on blinded specimens by a trained pathologist who scored all lesions on one lung section for each animal in the experiment. Treatment of animals. To create a high type I IFN environment and to exacerbate disease in WT B6 mice, mice were administered pICLC intranasally (i.n.) twice a week $(6 \mu \mathrm{g}$ in $30 \mu \mathrm{l})$, as previously described ${ }^{15}$. For host-directed therapy mice were given PGE2 i.n. twice a week $(2 \mu \mathrm{g}$ in $30 \mu \mathrm{l})$ or zileuton (Zyflo CR, Cornerstone Therapeutics, Cary, NC) in drinking water ad libitum $\left(6 \mathrm{mg} \mathrm{ml}^{-1}\right)$ or both starting one day after infection, unless stated otherwise. For antibiotic treatment mice were fed by gavage daily with isoniazid $(625 \mu \mathrm{g})$, rifampicin $(250 \mu \mathrm{g})$ and pyrazinamide $(3.75 \mathrm{mg})$ during the indicated time period.

Clinical cohort description for Chennai, India. Baseline plasma samples were originated from clinical protocols approved by the Institutional Review Board (IRB) of the National Institute for Research in Tuberculosis (NIRT), NCT01154959 and NCT00342017. Written informed consent was obtained from all participants or their legally responsible guardians and all clinical investigations were conducted 
according to the principles expressed in the Declaration of Helsinki. Samples were collected from a cohort of 97 subjects with active pulmonary TB (PTB), 14 individuals with latent TB infection (LTBI) and 20 healthy controls (HC) recruited as part of a TB cohort study at the Government Stanley Medical Hospital and at TB clinics supported by the National Institute for Research in Tuberculosis (NIRT), Chennai, India and described previously ${ }^{31}$.

TB diagnosis was based on culture positivity of sputum smears. Three sputum per subject were examined by fluorescence microscopy, processed by the modified Petroff s method and cultured on Lowenstein-Jensen medium. Positive cultures of M. tuberculosis were graded as $1+(20-100$ colonies), $2+$ (more than 100 discrete colonies) and $3+$ (more than 100 colonies forming a confluent mass). A posteroanterior chest X-ray was performed determine the extent of lung disease (unilateral vs. bilateral lesions), which was scored by three independent physicians from NIRT. LTBI diagnosis was based on tuberculin skin test (TST) and Quantiferon TB-gold ELISA positivity, absence of chest radiography abnormalities or pulmonary symptoms and negative sputum smears and cultures. HC individuals were asymptomatic with normal chest radiographies and negative sputum smears, cultures, Quantiferon results $(<0.35)$ and TST induration $(<5 \mathrm{~mm}$ in diameter). A time of enrolment, all individuals were HIV-negative, ATT-naive, BCG-vaccinated and had no record of prior TB disease. All individuals were negative for type-2 diabetes on the basis of glycosylated haemoglobin (HbAlc) levels and random blood glucose, according to the American Diabetes Association criteria (HbAlc levels $>6.5 \%$ and random plasma glucose $>200 \mathrm{mg} \mathrm{dl}^{-1}$ ). Haematology was performed on all individuals using the Act-5 Diff-hematology analyser (Beckman Coulter). Plasma levels of C-reactive protein (CRP) were assessed using a Luminex-based ELISA system (Bio-Rad, Hercules, CA). Study groups were similar with regard to age and gender and a summary of the characteristics is shown in Supplementary Tables 1 and 2 where the patients with active TB were compared according to the sputum smear positivity. Individuals with positive sputum smears had higher CRP values in plasma and presented more frequently with bilateral lung disease than those with negative smears.

Clinical cohort description for Zhengzhou, China. Persons with signs and symptoms indicative of active $\mathrm{TB}$ and who were $\mathrm{HIV}^{-}$and administered less than two weeks of anti-tubercular chemotherapy or community controls were enrolled into a prospective clinical protocol to assess response to chemotherapy (NCT01071603) conducted at the Henan Chest Hospital (HCH) from 2010 to 2012. IRBs from HCH and US National Institute for Allergy and Infectious Disease (Bethesda, Maryland) reviewed and approved the protocol and written informed consent was obtained from all participants. The protocol was conducted according to the ethical principles embodied in the Declaration of Helsinki and local and national Chinese regulations.

Participants described here were enrolled into one of the two cohorts for those with pulmonary tuberculosis (sputum AFB smear ${ }^{+}$cohort or the sputum AFB smear ${ }^{-}$cohort) based on the hospital's initial results. The enrolled subjects all underwent a chest CT, provided three sputum samples for AFB smear and culture by both the BACTEC MGIT 960 system (Becton, Dickenson and Company) and Lowenstein-Jensen medium (Chuang Xin Company; Hangzhou, China), and had blood drawn for routine blood chemistry and cytology as well as several experimental assays at enrolment. The $\mathrm{HCH}$ radiology department provided a scored assessment of the chest CTs that included locations of disease by lobe, type of abnormalities and number of cavities for each CT. The sputum smears were prepared by the ZeilNeilsen method using $1 \%$ carbolfuchsine $e^{32}$ and scored using the International Union Against Tuberculosis and Lung Disease (IUATLD) scale. The 94 subjects included in this analysis of the two PTB cohorts were confirmed to match their cohort definition, and to have abnormal chest CT scans consistent with active PTB. Fifteen percent of the subjects had a history of treatment for TB and less than $5 \%$ had a history of diabetes. Healthy community controls were recruited from Zhengzhou, China and included hospital personnel but excluded persons known to have a history of tuberculosis or HIV infection. Each enrolee was given a physical examination, a chest $\mathrm{CT}$, and the same microbiologic, biochemical and cytology tests administered to the patient cohorts. Those HC and LTBI participants presented in this analysis lacked radiologic and microbiological signs of active M. tuberculosis infection and had no pulmonary symptoms of tuberculosis, 14 individuals were found to have latent TB infection as indicated by a positive Quantiferon Gold in-the-tube test result and the remaining 11 had a negative result. BCG vaccination is administered in China and the majority of trial enrolees had a scar from vaccination so the TST was not administered. Haematology was performed on all individuals using the Sysmex Xt-1800i (Sysmex co., LTD, Japan). Plasma levels of C-reactive protein (CRP) were assessed using an Olympus Chemistry Analyzer (Beckman Coulter) and the Nanopia CRP kit (Sekisui Medical Co. LTD, Japan). A summary of the patient characteristics of all study groups is shown in Supplementary Table 3. Supplementary Table 4 compares characteristics of subjects with active TB based on their sputum smear positivity. Supplementary Table 5 compares characteristics of patients within the $\mathrm{PTB}$ group that were further stratified into mild (AFB-, unilateral lung lesions) and severe disease ( $\mathrm{AFB} \geq 1+$, and bilateral lung lesions).

Statistical analysis of clinical data sets. Median values with interquartile ranges were used as measures of central tendency. First, anthropometric and haematological characteristics were compared between the groups of patients with different clinical categorizations (HC, LTBI and PTB) using the non-parametric KruskalWallis test (for continuous variables) or the chi-square test (for categorical variables) (Supplementary Table 1 for India, Supplementary Table 3 for China). Further analyses compared the distribution of the same anthropometric and haematological parameters among PTB patients with positive identification of acid fast bacilli (AFB) in sputum smears and those with negative smears using the Mann-Whitney test (for continuous variables) of the Fisher's exact test (for categorical variables) (Supplementary Table 2 for India, Supplementary Table 4 for China). Mann-Whitney or Fisher's exact tests, which compared distributions of parameters were used to infer clinical severity between PTB patients with unilateral lung lesions and negative sputum smears (mild TB) and those with bilateral lesions and positive smears (severe TB). This analysis was limited to the Chinese data set, since the Indian data set did not include all the clinical parameters required for this specific test (Supplementary Table 5).

Plasma concentrations of cytokines and lipid mediators were compared between clinical groups (HC, LTBI, PTB AFB - and PTB AFB +) using the Kruskal-Wallis test with the non-parametric Mann-Kendall linear trend post hoc comparison. This type of post hoc test was specifically used to assess the general trend of variation of the plasma values of each plasma factor according to the degree of infectious burden (Supplementary Table 6 and 7). A similar approach was used considering the exact AFB sputum grade, where upward and downward trends were observed depending on the threshold of AFB grade considered (Fig. 3b).

The distribution values for each plasma factor used are shown in Supplementary Table 6 for India and Supplementary Table 7 for China.

Canonical correlation analysis (CCA) modelling was employed to assess whether combinations of circulating factors of the IL-1, IFN and eicosanoids pathways can discriminate between patients that present different degrees of TB severity (Fig. 3a). The CCA model was chosen as the number of variables studied was relatively high (13 variables were investigated in 131 individuals from India and 119 from China). This model is able to perform dimensionality reduction for two co-dependent data sets (CC1 and CC2) simultaneously so that the discrimination of the clinical endpoints represents a combination of variables that are maximally correlated. Thus, trends of correlations between parameters in different clinical groups rather than their respective distribution within each group are the key components driving the discrimination outcome. In our CCA model, simplified and adapted from previously reported investigations of biomarkers for TB diagnosi ${ }^{28}$, linear regression graphs represent coefficients from different combinations of plasma factors. The combinations of parameters used were the ones resulting in maximum values for correlation analysis first in India, and then applied without modifications in the Chinese data set. In the $x$-axis, the canonical coefficient (CC1) values were composed with data from the following variables: IL- $1 \alpha$, IL- $1 \beta$, IFN- $\gamma$, IFN- $\beta$, IFN- $\alpha$, IL-10, sIL1R1, sIL1R2 and IL1Ra. In the $y$-axis, the variables considered for the canonical coefficient (CC2) were: PGF2 $\alpha$, PGE2, LXA4 and 15-LXA4 (Fig. 3a). The diagnostic class prediction values obtained for each study site are shown in Supplementary Table 8 and were calculated using receiver operator characteristics curve analysis. The statistical analyses were performed using JMP 10 (SAS, Cary, NC), STATA 10 (StataCrop LP, College Station, TX) and GraphPad Prism 6.0 (GraphPad Software, San Diego, CA).

Statistical analyses. The statistical significance of differences between data groups was determined using the Mann-Whitney test, Wilcoxon matched pairs test or the Mantel-Cox test using GraphPad Prism 6. Averages with s.d. are shown. ${ }^{*} P \leq 0.05$ $* * P<0.005 ; * * * P<0.0005$; significant differences compared as indicated in figure by lines or compared to WT control groups.

31. Andrade, B. B. et al. Plasma heme oxygenase-1 levels distinguish latent or successfully treated human tuberculosis from active disease. PLOS ONE 8 e62618 (2013).

32. Humason, G. L. in Animal Tissue Techniques Ch. 20, 355 (W. H. Freeman, 1979).

33. Redford, P. S. et al. Influenza A virus impairs control of Mycobacterium tuberculosis co-infection through a type I interferon receptor dependent pathway. J. Infect. Dis. 209, 270-274 (2014). 
a
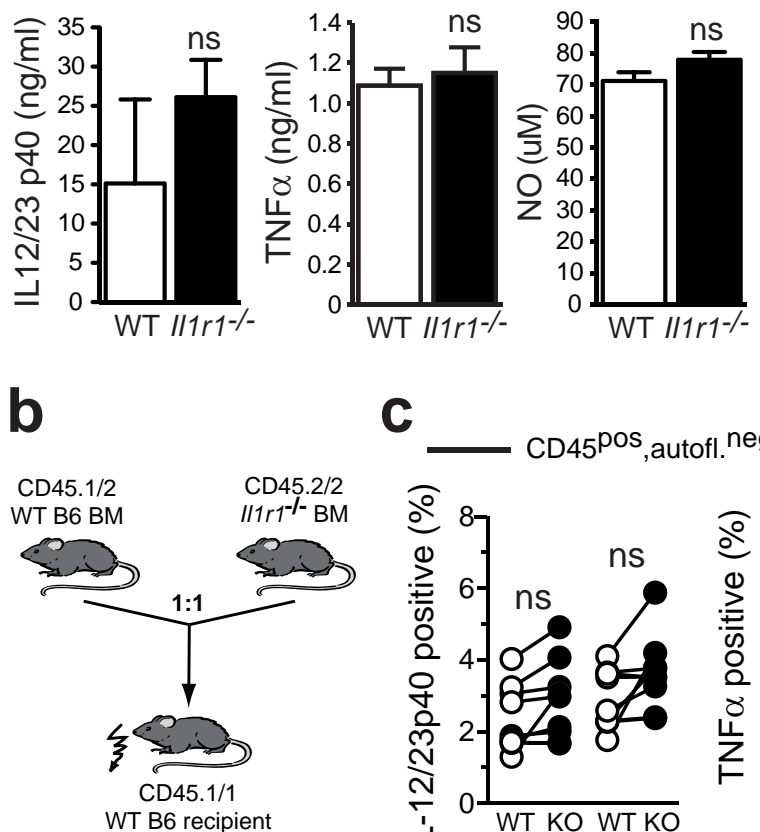

C

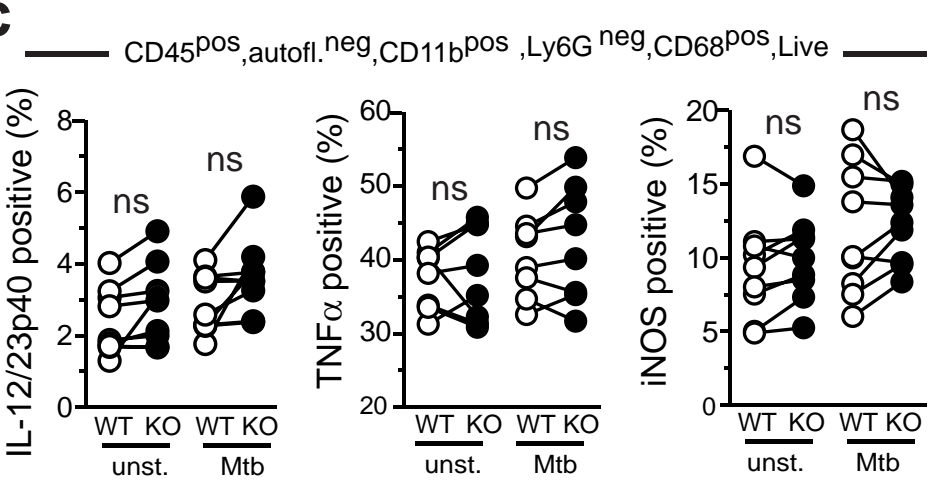

d
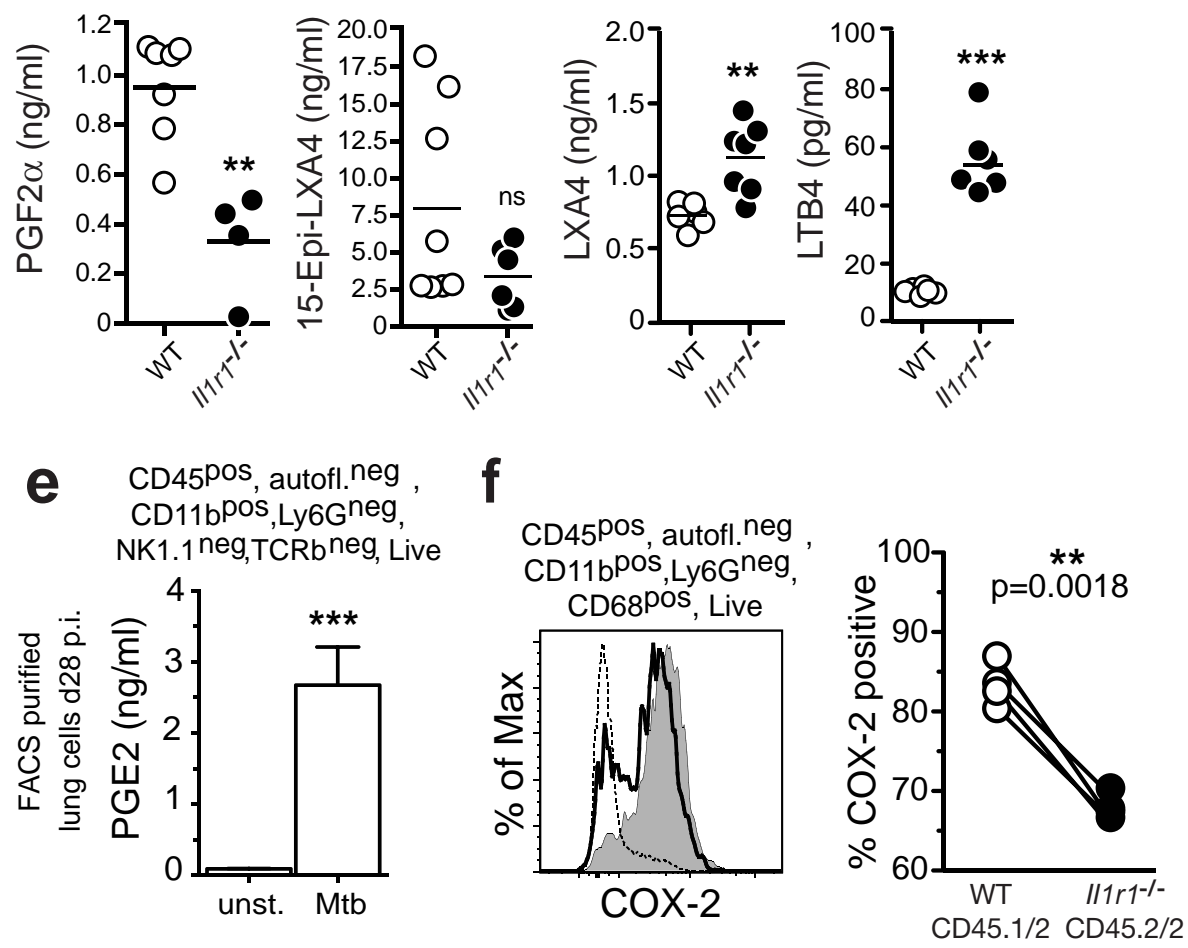

Extended Data Figure $1 \mid$ Lipid mediator production and COX2 expression in $I 11 \mathrm{r}^{-1-}$ animals and mixed bone-marrow chimaeric mice after Mtb infection. a, IL12/23 p40, TNF- $\alpha$ or NO measured in supernatants of WT and Illr1 $1^{-1-}$ BMDM after exposure to live Mtb (m.o.i. = 3). b, CD45.1/1 mice were lethally irradiated and reconstituted with equal ratios of WT (CD45.1/2) and $I l 1 r 1^{-1-}$ (CD45.2/2) bone-marrow cells and infected with Mtb.

c, Frequency of IL-12/23p40, TNF- $\alpha$ or iNOS expression in WT (white circles) or $I l 1 r 1^{-1-}$ (KO, dark circles) total $\mathrm{CD} 68^{\text {pos }}$ mononuclear myeloid cells, gated as indicated. Each connecting line depicts an individual animal. Data in are representative of two independent experiments with 3-5 mice each. Paired $t$-tests $P$ values are indicated (n.s., not significant). d, PGF $2 \alpha, 15$-Epi-LXA4, LXA4 or LTB4 concentrations in BALF 25 days after Mtb infection of WT or
Ill $11^{-/-}$mice. e, Pulmonary single-cell suspensions from day $28 \mathrm{Mtb}$-infected WT mice were FACS-sorted based on indicated surface marker expression and cultured for $16 \mathrm{~h}$, with (white bars) or without addition of Mtb (grey bars), after which PGE2 was measured in culture supernatants. f, Analysis of donor bone-marrow derived $\mathrm{CD} 68^{\text {pos }}$ myeloid cells 4 weeks p.i. in isolated lung cells marked by CD45.1 and CD45.2 expression for COX-2 protein expression (left, filled histogram: WT cells; thick line: $I l 1 r 1^{-1-}$ cells; dotted line: isotype control) and frequency (right) of COX-2 expression by WT (white circles) or Illr1 $1^{-1-}$ (dark circles) total CD68 ${ }^{\text {pos }}$ mononuclear myeloid cells. Each connecting line depicts an individual animal. AU, arbitrary units. Data are representative of two independent experiments with 3-5 mice each. Paired $t$-tests $P$ values are indicated (n.s., not significant). 
a

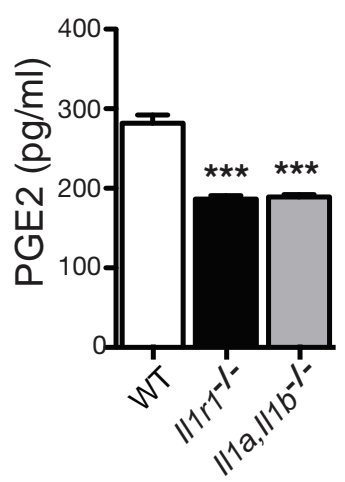

e

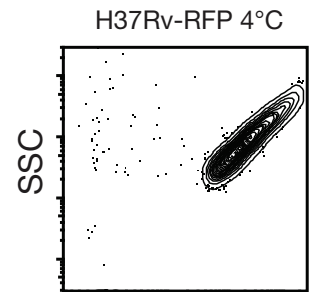

H37Rv-RFP

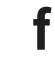

f

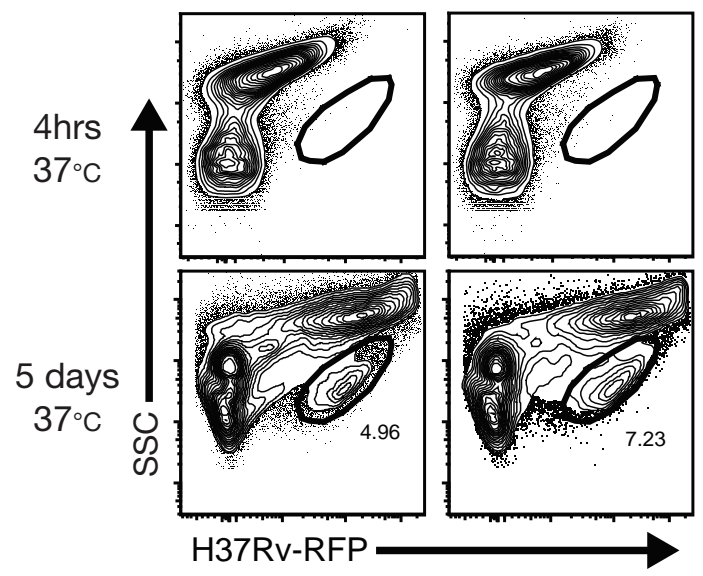

Extended Data Figure $2 \mid$ Characterization of extracellular bacilli in WT or IL-1-deficient macrophage cultures. a, PGE2 measured in supernatants of WT and Ill $r 1^{-1-}$ or Ill $\alpha, I l 1 \beta^{-1-}$ BMDM after exposure to live Mtb $(\mathrm{m}$. o.i. $=3)$. b, Ratio of PGE2 to LXA4 in WT or Illr1 ${ }^{-1-}$ BMDC $40 \mathrm{~h}$ after live Mtb (m.o.i. = 10) infection. WT, Illr1 $1^{-1-}$ or $I l 1 \alpha, I l 1 \beta^{-/-}$BMDM were infected with H37Rv Mtb (m.o.i. = 3) in vitro. c, d, 5 days later free extracellular bacilli in each culture was assessed through microscopy on cytospin slides (c) as well as the frequency of infected macrophages (d). 4-5 view fields, a total of 454 macrophages, per experimental group, were blindly scored. Data shown are the means \pm s.d. and are representative of 2 independent experiments. $* * * P \leq 0.0005$ significant differences compared to WT cells. e, H37Rv-RFP
C

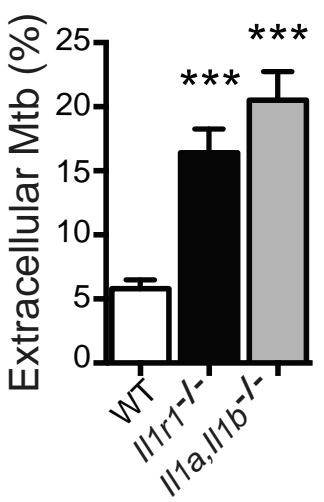

BMDM + H37Rv-RFP

xed $1: 1$ for 2 minutes at $4^{\circ} \mathrm{C}$
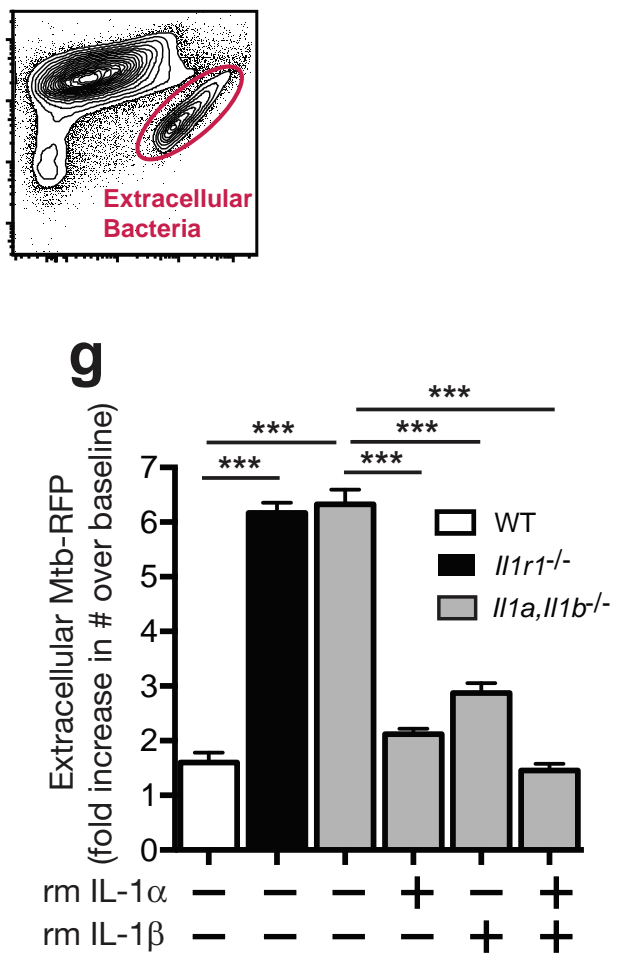

(left panel) alone, BMDM alone (middle panel) or H37Rv-RFP mixed briefly with BMDM (right panel) to quantify free extracellular bacteria by flow cytometry. f, WT or Illr1 ${ }^{-1-}$ BMDM were infected with H37Rv-RFP $(\mathrm{m}$. o.i. $=3)$ in vitro. Free bacteria in cultures were analysed by FACS at indicated time points. g, WT, Illr1 $1^{-/-}$or Ill $\alpha, I l 1 \beta^{-/-}$BMDM were infected with H37Rv-RFP (m.o.i. $=3$ ) in vitro and in the presence or absence of recombinant murine IL- $1 \alpha$, IL- $1 \beta$ or both. 5 days later cell-free bacteria were measured by FACS. $* * * P \leq 0.0005$ significant differences in indicated comparisons. Data shown are the means \pm s.d. and are representative of 2 independent experiments. 

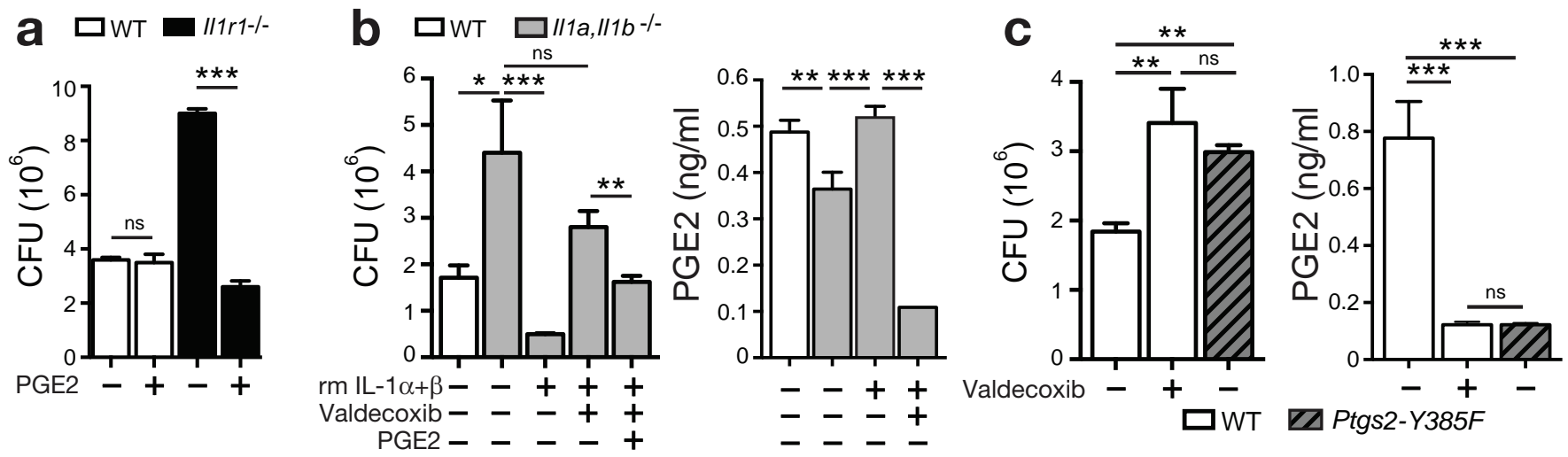

d
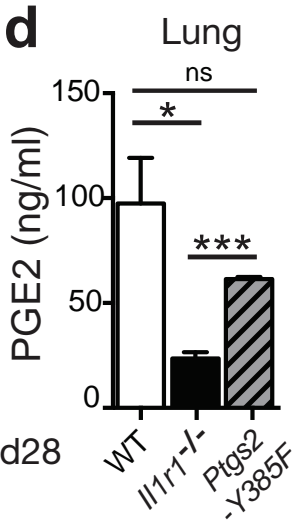

Serum
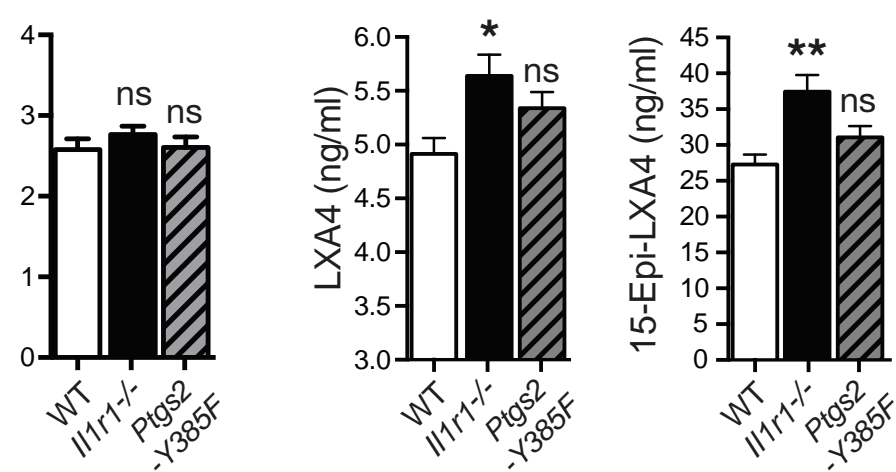

Extended Data Figure $3 \mid$ Role of COX-2 in IL-1-dependent PGE2 synthesis and bacterial control. a, c.f.u. at 5 days after in vitro infection (m.o.i. $=3$ ) of $\mathrm{WT}$ or Illr1 $1^{-1-}$ BMDM in the presence or absence of PGE2. b, c.f.u. at 5 days after in vitro infection (m.o.i. $=3$ ) of WT or Ill $\alpha, I l 1 \beta^{-/-}$BMDM in the presence or absence of IL1, PGE2 or the COX-2 inhibitor valdecoxib (left) and PGE2 concentrations after $48 \mathrm{~h}$ (right). c, c.f.u. at 5 days after in vitro infection (m.o.i. $=3$ ) of WT or Ptgs2(Y385F) enzymatic activity-deficient $\mathrm{BMDM}$ in the presence or absence of the COX-2 inhibitor valdecoxib (left) and PGE2 concentrations after $48 \mathrm{~h}$ (right). d, PGE2 concentration in lungs (left)

and serum (right) of 4-week Mtb-infected WT, Il1r1 ${ }^{-1-}$ or ptgs2(Y385F) enzymatic activity-deficient animals. e, LXA4 and 15-Epi-LXA4

concentrations or in serum of 4 weeks. Infected WT, Illr1 $1^{-1-}$ or ptgs2(Y385F) enzymatic activity-deficient animals after aerosol challenge with $100-150$ c.f.u. H37Rv. $* P \leq 0.05$ and $* * P \leq 0.005$, significant differences compared to WT. Data shown are the means \pm s.d. and are representative of 2 independent experiments. e, IL-1 $\beta$ concentration in supernatant of primary human monocyte derived macrophages from 35 healthy donors, $24 \mathrm{~h}$ after Mtb infection (m.o.i. $=5)$ in relation to LDH levels in same cultures. 
a

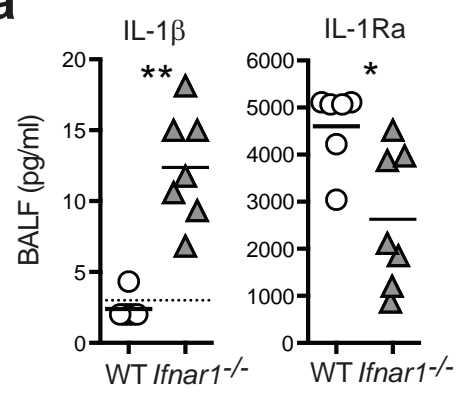

b

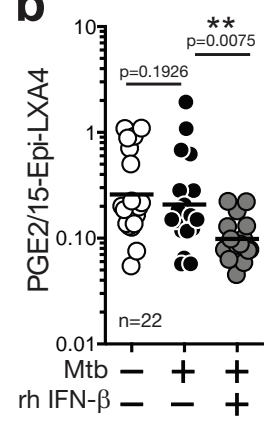

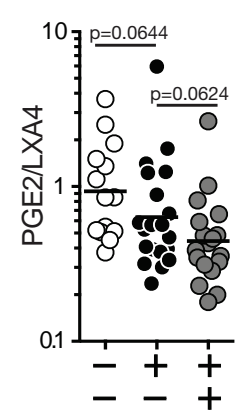

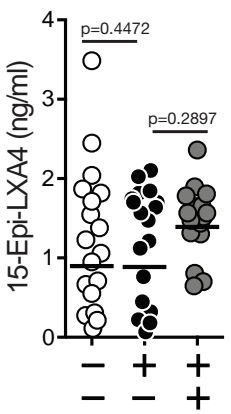

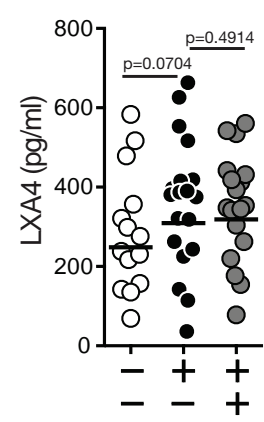

C

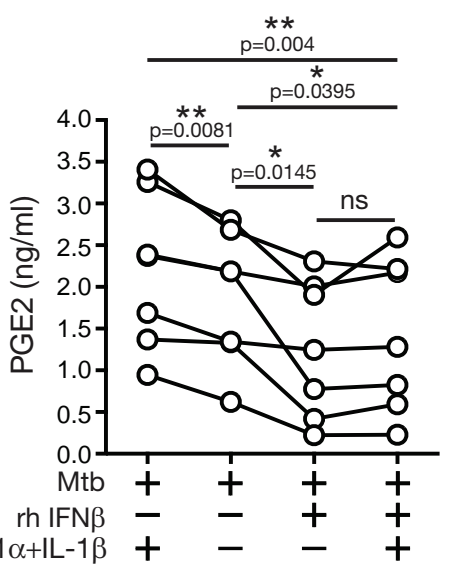

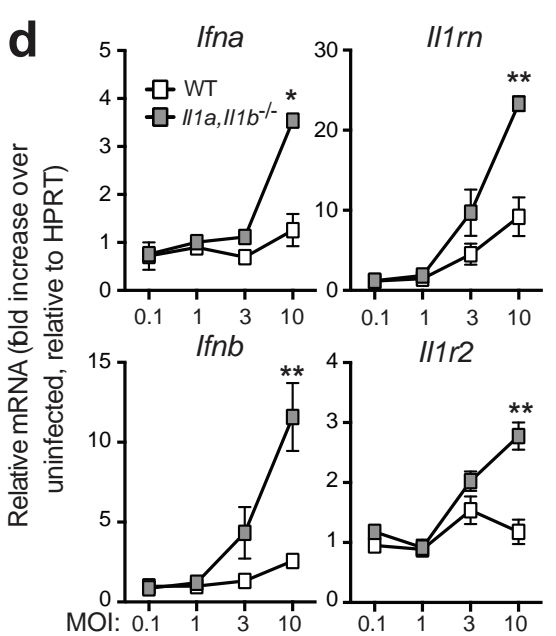

e
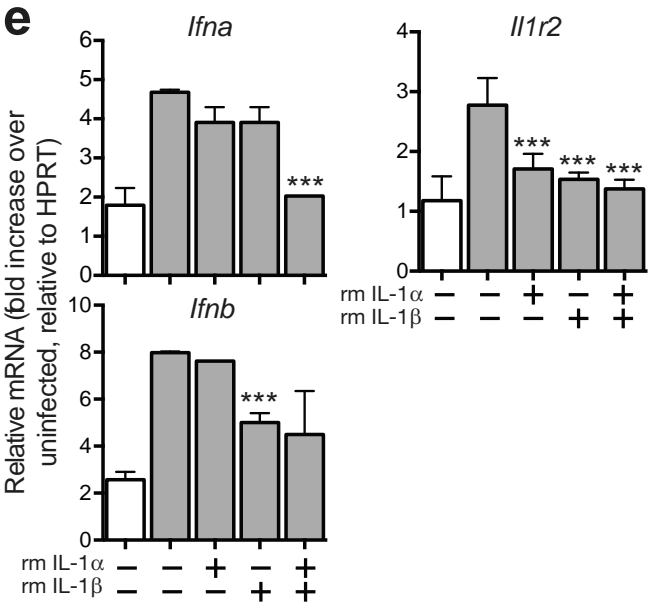

Extended Data Figure $4 \mid$ IL-1 type I IFN crosstalk. a, IL-1 $\beta$ and IL1Ra concentrations measured in BALF of WT or Ifnar $1^{-/-}$mice 4 weeks p.i. b, PGE2/15-Epi-LXA4 and PGE2/LXA4 ratios, LXA4, and 15-Epi-LXA4 concentrations in supernatants of primary human MDM from 22 healthy donors, $24 \mathrm{~h}$ after Mtb infection (m.o.i. $=5$ ) in the presence or absence of rhIFN- $\beta$. Differences were compared as indicated by lines. c, PGE2 concentrations in culture supernatants of primary human MDM from 7 healthy donors, $24 \mathrm{~h}$ after Mtb infection (m.o.i. = 5) in the presence or absence of rhIFN- $\beta$, rhIL- $1 \alpha$ and Il-1 $\beta$ or PGE2. Differences were compared as indicated by lines. d, WT (white) or Ill $\alpha$, Ill $\beta^{-/-}$(light grey) BMDM were infected with increasing m.o.i. of Mtb in vitro. mRNA expression of indicated genes was determined at 40 h. e, WT (white) or Ill $\alpha, I l 1 \beta^{-/-}$(light grey) BMDM were infected with m.o.i. $=3$ of $\mathrm{Mtb}$ in vitro in the presence or absence of recombinant murine IL- $1 \alpha$, IL- $1 \beta$ or both. mRNA expression of indicated genes was determined at $40 \mathrm{~h}$. Data shown are the means \pm s.d. and are representative of 2 independent experiments. 


\section{RESEARCH LETTER}
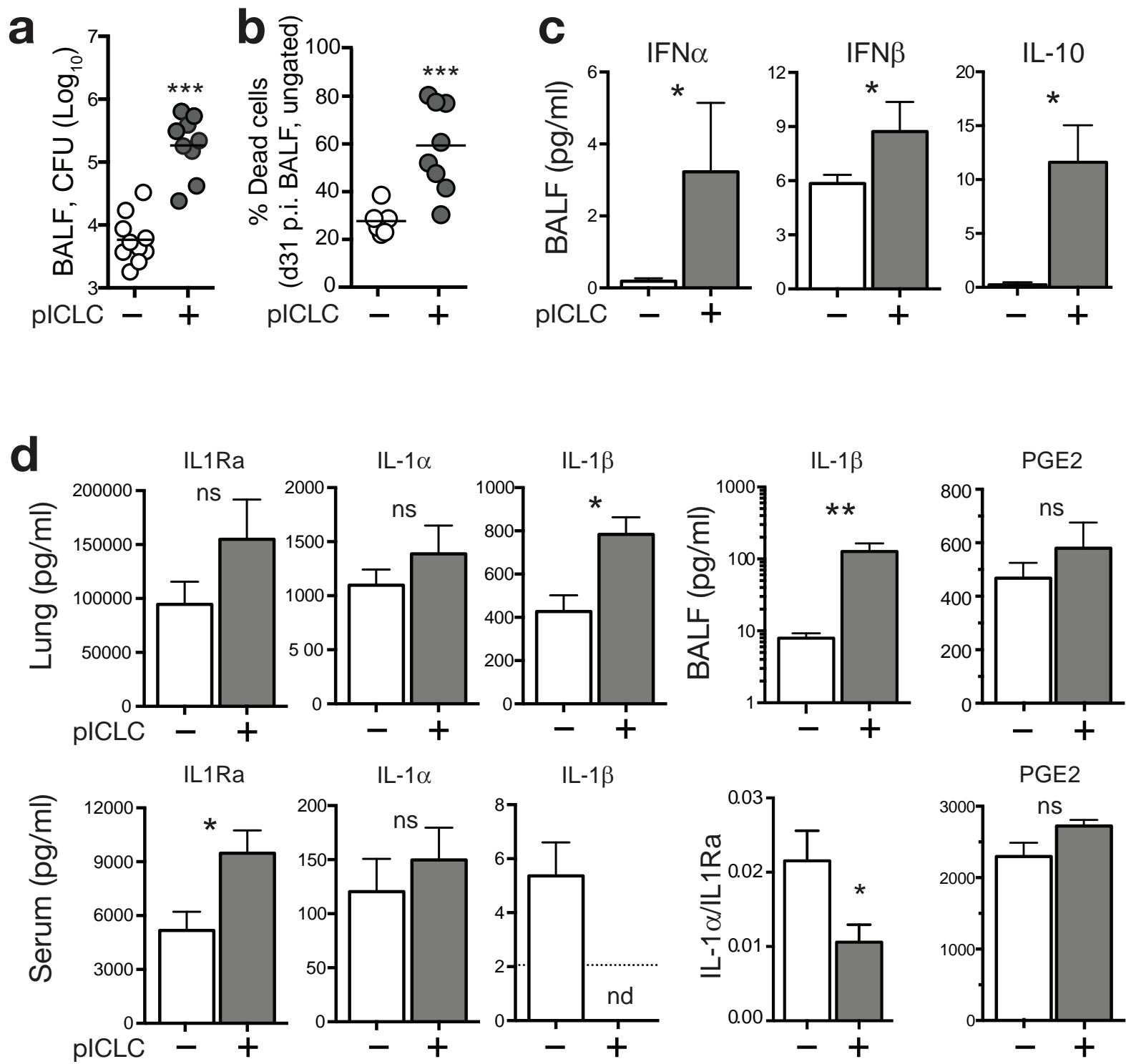

Extended Data Figure $5 \mid$ Cytokine expression in Mtb-infected pICLCtreated WT mice. WT mice were treated with $\operatorname{pICLC}(+)$ or without $(-, \mathrm{PBS})$ intranasally twice a week, starting day 1 after aerosol challenge with Mtb. in BALF at 4 weeks post infection (c). d, Indicated cytokines and eicosanoids were measured by ELISA in lung homogenates, BALF and serum 4 weeks p.i. Data are representative of a minimum of 3 independent experiments with a a-c, c.f.u. (a), FACS analysis of dead cells (b) and indicated cytokines measured

minimum of 4 mice per group. 

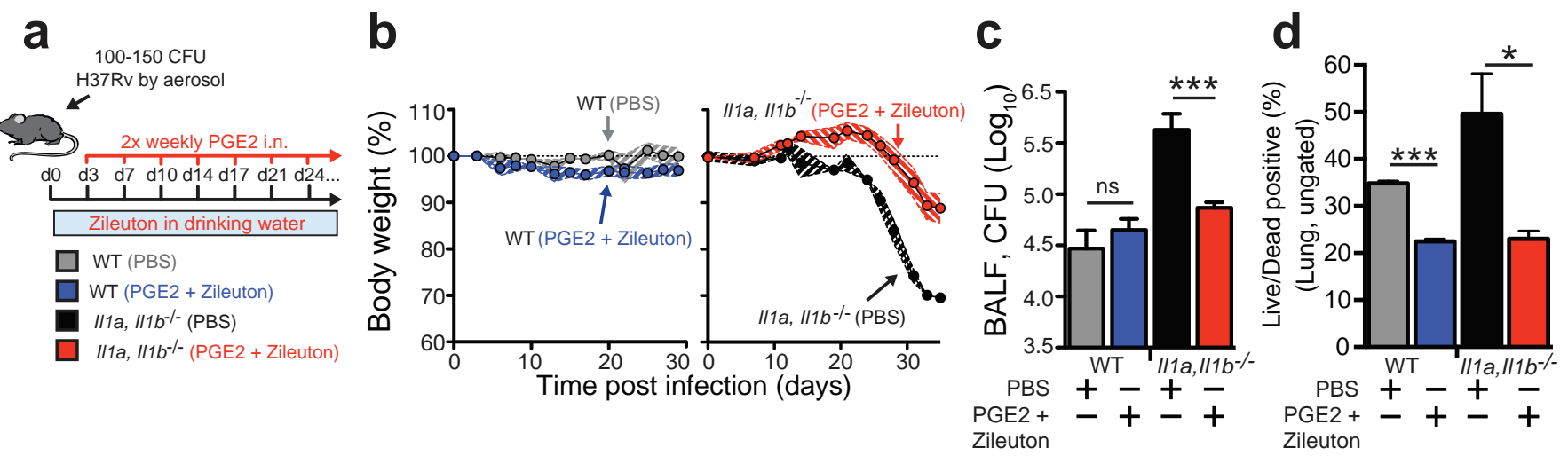
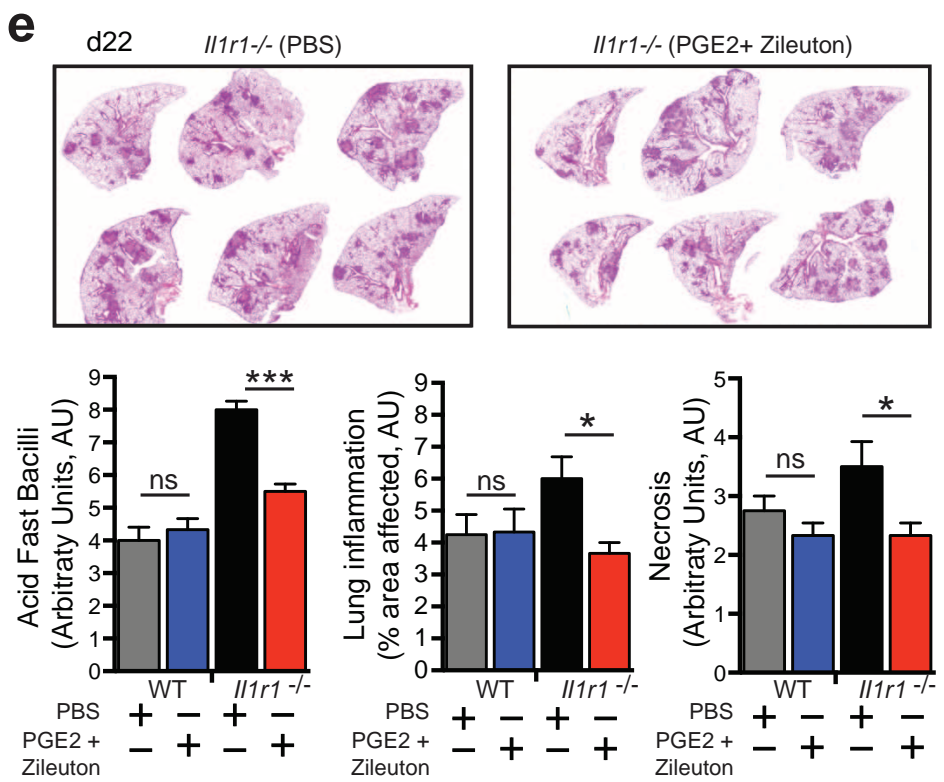
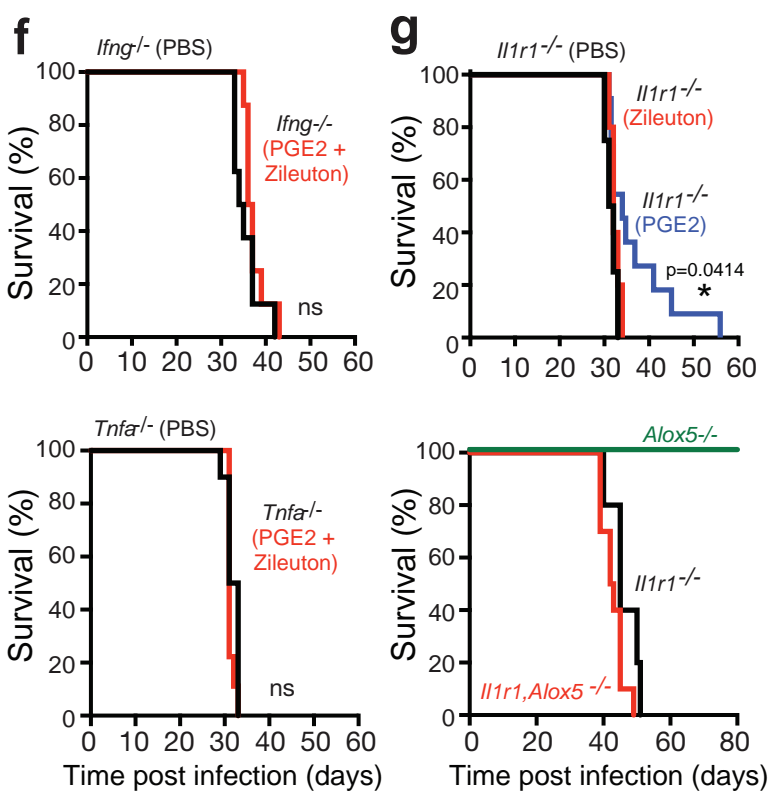

Extended Data Figure $6 \mid$ PGE2 administration and 5-LO inhibition in IL1-deficient animals reduces necrotic pathology and increases bacterial control. a, Experimental design for host-directed therapy with PGE2 and zileuton in Mtb-infected mice. b, Weight loss (travelling error bars indicate s.d., $n=6$ ) during Mtb infection of WT (left) or Ill $\alpha, I l 1 \beta^{-1-}$ (right) mice treated with or without i.n. PGE2 and zileuton. $\mathbf{c}, \mathbf{d}$, Bacterial loads in BALF (c) or FACS analysis (d) of dead cells 21 days after aerosol exposure to Mtb (H37Rv) in lungs of WT or $I l 1 \alpha, I l 1 \beta^{-l-}$ treated with or without i.n. PGE2 and zileuton. e, Representative haematoxylin-and-eosin-staining of lung lobes of untreated Illr1 $1^{-/-}$or treated $I l 1 r 1^{-/-}$with i.n. PGE2 and zileuton in drinking water (top panels). Bottom panels show the relative number of acid fast bacilli, lung inflammation (\% lung affected, mean granuloma sizes) and degree of necrosis scored blindly by a trained pathologist. Differences were compared as indicated by lines. Data shown are representative of two independent experiments with a minimum of 6 mice per group. f, Survival after aerosol exposure to Mtb (H37Rv) of Ifng ${ }^{-1-}$ (top panel) or Tnfa ${ }^{-1-}$ (bottom panel) untreated (PBS) or treated i.n. PGE2 (twice a week) and zileuton (in drinking water) starting at day 1 p.i. Data shown are representative of three independent experiments with a minimum of 4 mice per group. g, Survival after aerosol exposure to Mtb $(\mathrm{H} 37 \mathrm{Rv})$ of $I l 1 r 1^{-1-}$ (top panel) treated with either PGE2 or zileuton. Data shown are representative of two independent experiments with a minimum of 4 mice per group. Bottom panel shows survival of $I l 1 \mathrm{rl}^{-1-}$, Alox $5^{-1-}$ or Illr1,Alox $5^{-/-}$mice. Data shown are representative of three independent experiments with a minimum of 4 mice per group. 

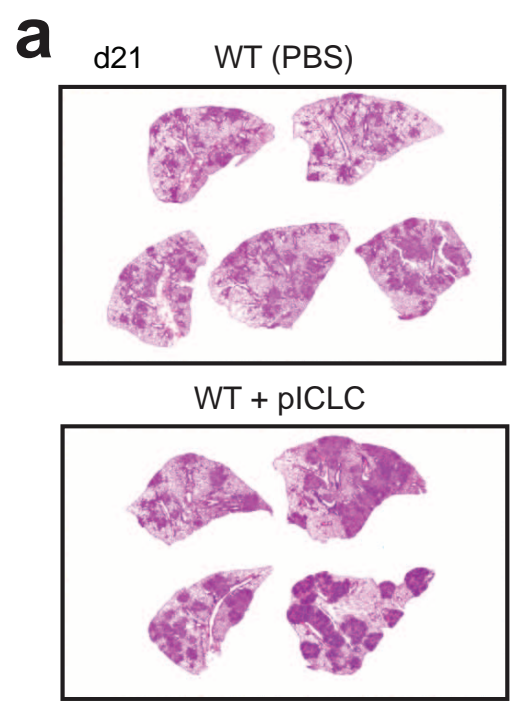

WT + pICLC + PGE2+ Zileuton
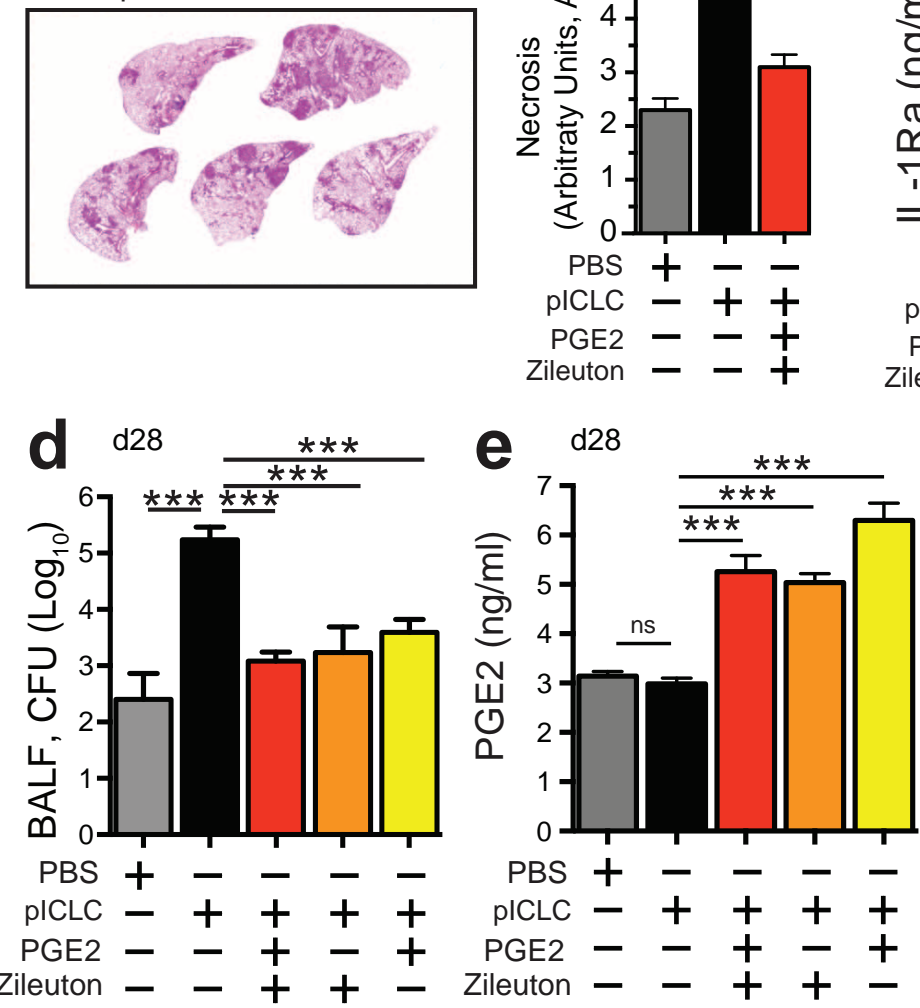
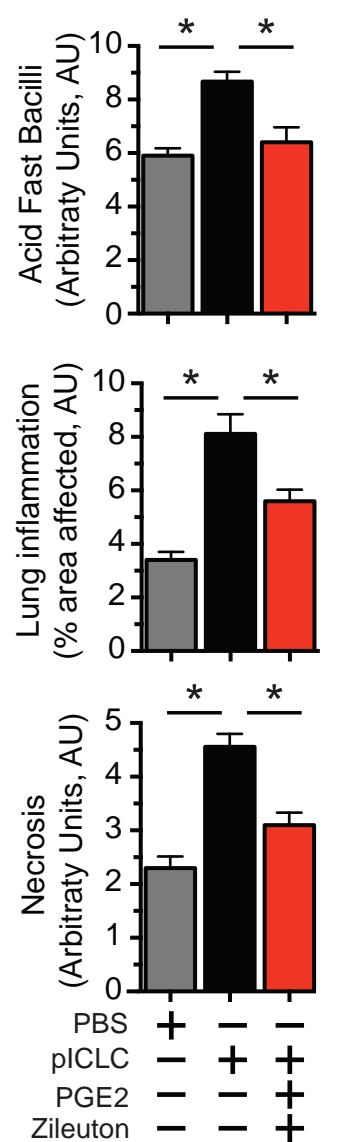
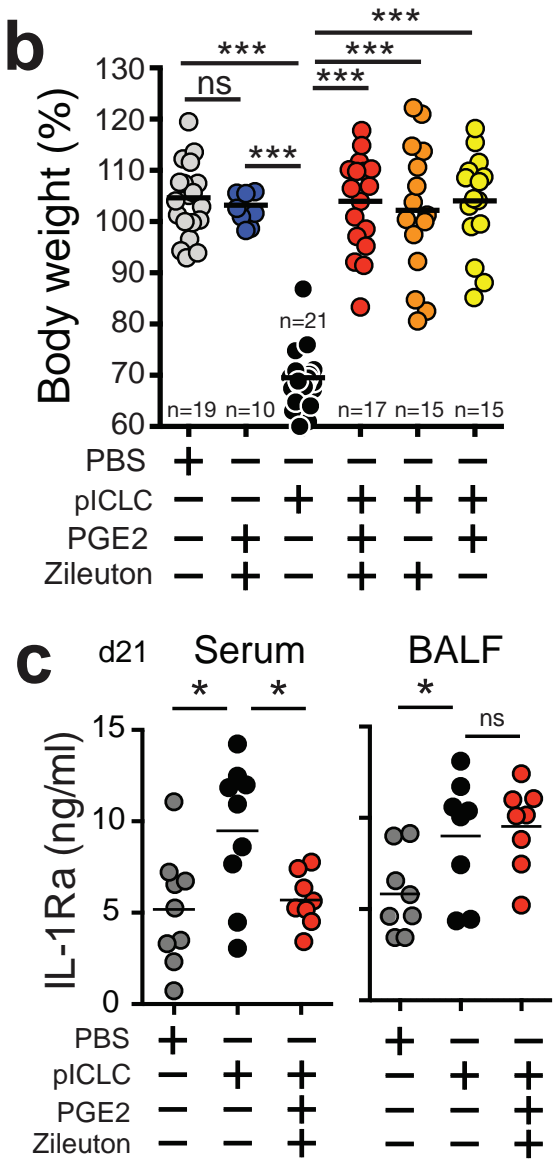

Extended Data Figure $7 \mid$ PGE2 and/or 5-LO inhibition in pICLC-treated WT mice reduces necrotic pathology and increases bacterial control. a, WT mice were treated with pICLC or without (PBS) intranasal twice a week, starting day 1 after aerosol challenge with Mtb. Representative haematoxylin-andeosin-staining of lung lobes of untreated WT (PBS), pICLC-treated or pICLCtreated with i.n. PGE2 and zileuton in drinking water (left panels) three weeks after Mtb infection. Right panels show the relative number of acid fast bacilli, lung inflammation (\% lung affected, mean granuloma sizes) and degree of necrosis scored blindly by a trained pathologist. Differences were compared as indicated by lines. Data shown are representative of two independent experiments with a minimum of 4 mice per group. $\mathbf{b}$, WT mice were treated as indicated in figure. Weight loss in experimental groups was normalized to time of moribundity of last remaining mouse in pICLC group (between day 40 and day 100, depending on experiment). For pICLC group (black), weight loss is shown for each animal at time of moribundity. Data shown are combined from three individual experiments with 3-7 mice per group. Differences were compared to pICLC (black) group. c, WT mice were untreated (PBS) treated with pICLC or pICLC, PGE2 and zileuton intranasally starting day 1 after aerosol challenge with Mtb. 3 weeks post infection IL1Ra was measured by ELISA in BALF and serum. Data shown are representative of two independent experiments with a minimum of 4 mice per group. d, Bacterial loads 28 days after aerosol exposure to Mtb (H37Rv) in BALF of pICLC-treated WT (black) mice with or without i.n. PGE2 (yellow) or zileuton (orange) or a combination of both (red) as indicated in figure. e, PGE2 and IFN- $\beta$ concentrations in BALF of indicated mice 4 weeks p.i. $* * * P \leq 0.0005$, significant differences compared to pICLC (black)-treated group. Data shown are representative of three independent experiments with a minimum of 4 mice per group. 
a

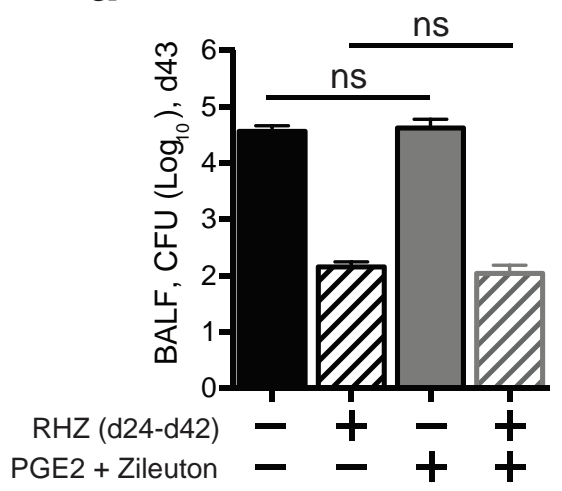

b

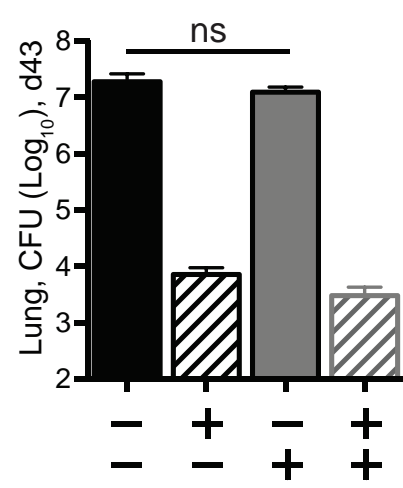

Extended Data Figure $8 \mid$ Host-directed therapy with PGE2 and zileuton does not interfere with antibiotic treatment. Bacterial loads in $\mathrm{C} 3 \mathrm{HeB} / \mathrm{FeJ}$ mice 43 days p.i. with $100-150$ c.f.u. H37Rv, treated or untreated with PGE2 intranasally and zileuton in drinking water. Starting day 24 p.i. antibiotic treatment with rifampicin, pyrazinamide and isoniazid was given $5 \times$ a week until one day before collection. a, b, Bacterial loads in BALF (a) and lungs (b). Differences were compared as indicated by lines. Data shown are from one experiment with 5-7 mice per group. 

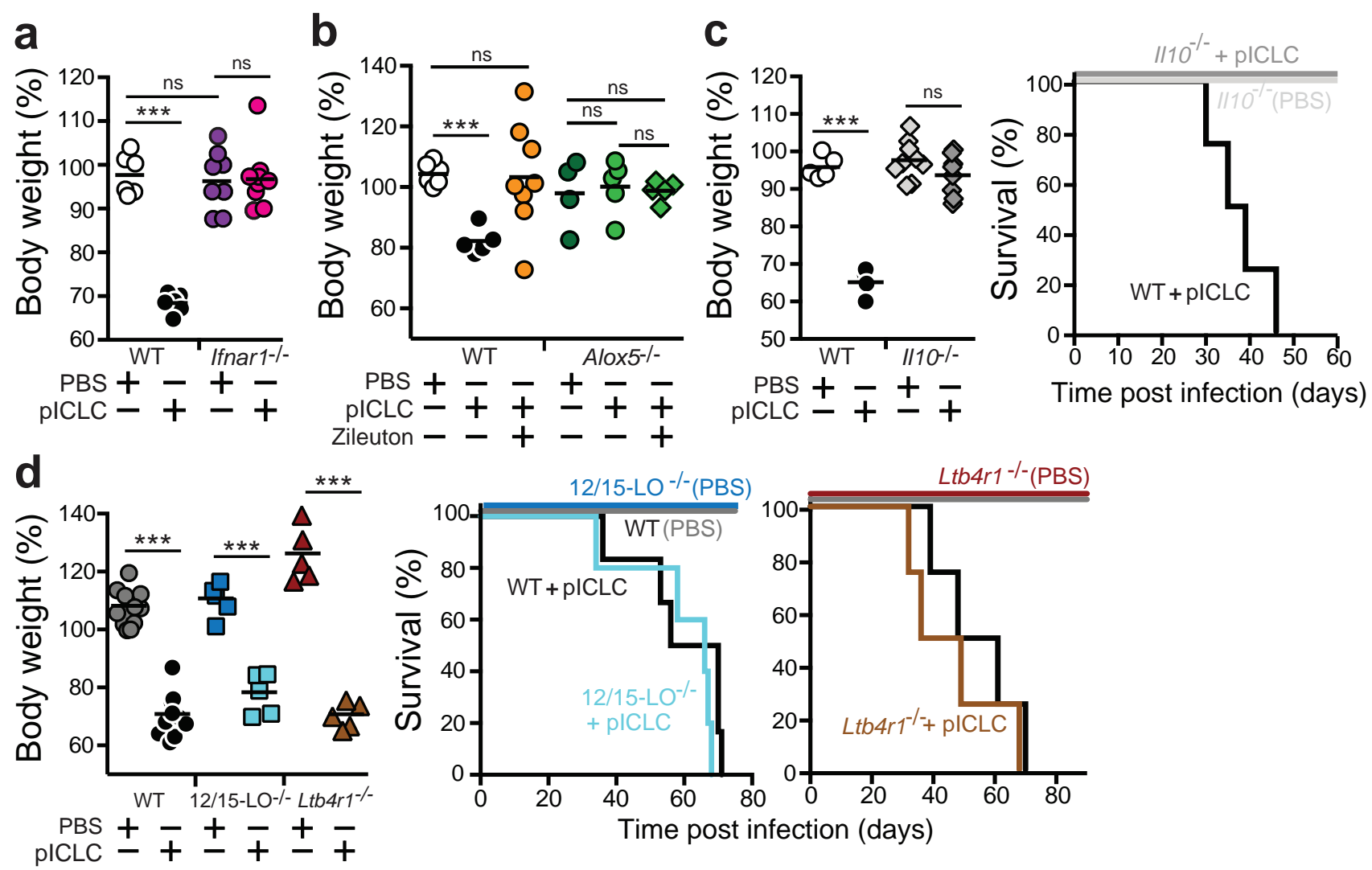

Extended Data Figure $9 \mid$ IL-10, but not LTB4 or 12/15-LO, is required for type I IFN-driven disease exacerbation of pICLC-treated WT animals infected with Mtb. a, WT or Ifnar $1^{-1-}$ mice were treated with pICLC or without (PBS) intranasal twice a week, starting day 1 after aerosol challenge with Mtb. Weight loss in experimental groups was normalized to time of moribundity of last remaining mouse in pICLC group (black) for each independent experiment. For pICLC group (black), weight loss is shown for each animal at time of moribundity. Data shown are combined from two individual experiments with 3-7 mice per group. Differences were compared as indicated by lines. b, WT or Alox $5^{-/-}$mice were treated with pICLC or without (PBS) intranasally twice a week or administered zileuton in the drinking water. Data shown are representative of two individual experiments with 4-8 mice per group. Differences were compared as indicated by lines. c, WT or $I l 10^{-/-}$mice were treated with pICLC or without (PBS) intranasally twice a week. For pICLC group (black), weight loss is shown for each animal at time of moribundity.
Data shown are representative of two individual experiments with 3-8 mice per group. Differences were compared as indicated by lines. Survival (right panel) of WT or $I l 10^{-1-}$ mice treated with (dark grey lines) or without (light grey lines). Data shown are representative of two individual experiments with 4-6 mice per group. d, WT, $12 / 15-\mathrm{LO}^{-/-}, \mathrm{Lt} b 4 r 1^{-/-}$mice were treated with pICLC or without (PBS) intranasally twice a week. For pICLC groups, weight loss is shown for each animal at time of moribundity. Data shown are representative of two individual experiments with 5-7 mice per group. Differences were compared to PBS controls in each mouse strain. Survival of WT or 12/15$\mathrm{LO}^{-/-}$mice (middle panel) treated with pICLC (dark blue lines) or without (light blue lines). Data shown are representative of two individual experiments with 4-6 mice per group. Survival of WT or $L t b 4 r 1^{-1-}$ mice (right panel) treated with pICLC (burgundy lines) or without (brown lines). Data shown are representative of two individual experiments with 4-6 mice per group. 
a

Inflammatory Equilibrium

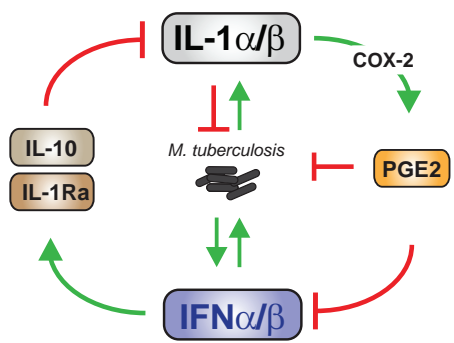

WT B6 mice:

(models latent state in TB patients)
High Type I IFN environment-

Scenario A: IL-1 deficiency

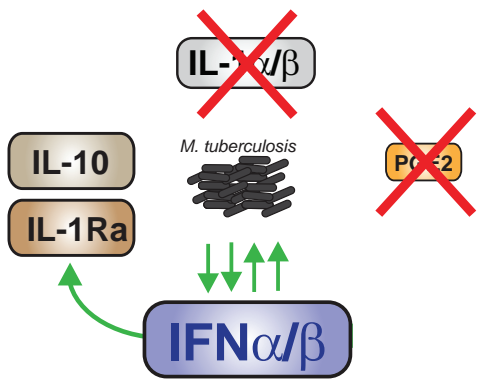

IL-1 deficient mice:

(models uncontrolled active disease in subset of TB patients)
HDT targeting PGE2:

Return to non-pathological equilibrium

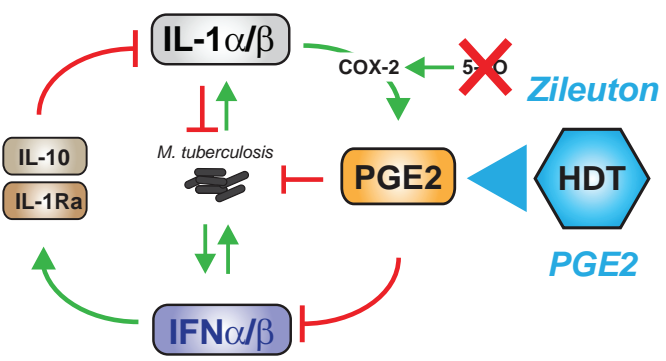

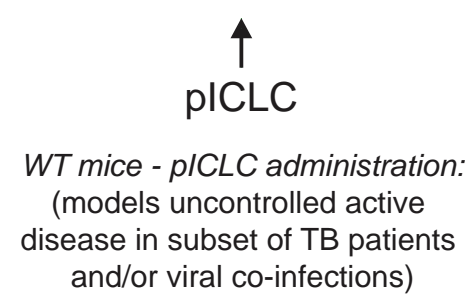

Extended Data Figure $10 \mid$ Schematic summary of IL-1-type I IFN counterregulation during Mtb infection. a, Virulent Mtb directly induces IL- $1 \alpha$ and IL- $1 \beta$ as well as type I IFNs in myelophagocytic cells. Whereas IL-1 is required for bacterial control, type I IFNs are considered detrimental because they can exert pro-bacterial effects. During low-dose aerosol infection in WT B6 mice the balance of these two pathways establishes an inflammatory equilibrium that allows for containment of bacilli and chronic infection and thus models certain aspects associated with latency in TB patients. This is achieved by an antagonist relationship whereby type I IFNs inhibit IL- $1 \alpha / \beta$ directly as well as through induction of IL-10 and IL1Ra, while in the opposing direction IL- $1 \alpha / \beta$ limit type I IFNs through COX-2-mediated PGE2 induction. $\mathbf{b}$, In the absence of IL1 , PGE2 fails to inhibit type I IFNs and the equilibrium is disturbed. This leads to excessive type I IFN expression that in turn causes increased bacterial replication and pathology, thus modelling the type I IFN-associated uncontrolled active disease that occurs in a subset of TB patients ${ }^{20-22}$. c, Likewise, experimental induction of type I IFNs by pICLC or viral coinfections ${ }^{33}$ creates a detrimental environment with uncontrolled bacterial growth and extracellular bacteria, ultimately resulting in loss of host resistance and increased mortality. d, HDT targeting PGE2, either directly or indirectly by enhancing PGE2 levels via 5-LO inhibition with zileuton, returns the system to a non-pathological steady state by limiting type I IFN-driven disease exacerbation, bacterial replication and pulmonary necrosis. Green arrows indicate inductive and red lines inhibitory pathways. 